\title{
A Robust Vision-based Runway Detection and Tracking Algorithm for Automatic UAV Landing
}

\author{
Thesis by \\ Khaled Abu Jbara
}

In Partial Fulfillment of the Requirements

For the Degree of

Masters of Science

King Abdullah University of Science and Technology, Thuwal,

Kingdom of Saudi Arabia

April, 2015 
The thesis of Khaled Abu Jbara is approved by the examination committee

Committee Chairperson: Ganesh Sundaramoorthi

Committee Member: Jeff Shamma

Committee Member: Christian Claudel 
Copyright (C)2015

Khaled Abu Jbara

All Rights Reserved 


\title{
ABSTRACT
}

\section{A Robust Vision-based Runway Detection and Tracking}

\author{
Algorithm for Automatic UAV Landing \\ Khaled Abu Jbara
}

This work presents a novel real-time algorithm for runway detection and tracking applied to the automatic takeoff and landing of Unmanned Aerial Vehicles (UAVs). The algorithm is based on a combination of segmentation based region competition and the minimization of a specific energy function to detect and identify the runway edges from streaming video data. The resulting video-based runway position estimates are updated using a Kalman Filter (KF), which can integrate other sensory information such as position and attitude angle estimates to allow a more robust tracking of the runway under turbulence. We illustrate the performance of the proposed lane detection and tracking scheme on various experimental UAV flights conducted by the Saudi Aerospace Research Center. Results show an accurate tracking of the runway edges during the landing phase under various lighting conditions. Also, these results suggest that such positional estimates would greatly improve the positional accuracy of the UAV during takeoff and landing phases. The robustness of the proposed algorithm is further validated using Hardware in the Loop simulations with diverse takeoff and landing videos generated using a commercial flight simulator. 


\section{ACKNOWLEDGEMENTS}

I am grateful to my mentor and guide, Prof. Ganesh Sundaramoorthi, for his tremendous support and unstoppable feedback during my research journey. Also, I would like to thank Prof. Jeff Shamma, and Prof Christian Claudel for being a part of the thesis committee and for their useful feedback about this thesis.

A special thanks to the Aerospace Division of King Abdulaziz City for Science and Technology (KACST) for supporting with experimental flight videos conducted by "SAKER 4" UAV.

In addition to that, I express my gratitude to the persons who experienced with me the hard time during the preparation of my master degree. I start with Lama Affara, whom first I should thank, for her generous technical support and for being very supportive and close friend. Moreover, Adel Bibi, is a real friend and scientist who kept encouraging me and discussing my progress.

Finally and simply, I designate the biggest gratitude for being successful in my life to my beloved Mother. 


\section{TABLE OF CONTENTS}

\begin{tabular}{ll}
\hline Examination Committee Approval & 2
\end{tabular}

\begin{tabular}{ll} 
Copyright & 3 \\
\hline
\end{tabular}

\begin{tabular}{ll}
\hline Abstract & 4
\end{tabular}

\begin{tabular}{|l|l|}
\hline Acknowledgements & 5
\end{tabular}

\begin{tabular}{lr}
\hline Nomenclature & 8
\end{tabular}

Abbreviations . . . . . . . . . . . . . . . . . . . . . . . . . . . 9

List of Symbols . . . . . . . . . . . . . . . . . . . . . . . . . . 9

\begin{tabular}{ll}
\hline List of Figures & 10
\end{tabular}

$\begin{array}{lll}1 & \text { Introduction } & 11\end{array}$

1.1 Objectives and Contributions . . . . . . . . . . . . . . . . 15

1.2 Related Work . . . . . . . . . . . . . . . . . . . . . . . . . . . . 17

2 Algorithms Overview and Runway Mathematical Model Representation 20

2.1 Runway detection and tracking algorithms overview and structure . . 20

$2.2 \quad$ Generic Model Representation and Energy Optimization . . . . . . . 22

3 Image Processing and Tracking Algorithms 26

3.1 Detection Method . . . . . . . . . . . . . . . . . . . . 26

3.2 Local Optimization for Refined Segmentation . . . . . . . . . . . . . 30

3.2 .1 Computation of the Gradient of $E$. . . . . . . . . . . . . . . 32

3.3 Tracking . . . . . . . . . . . . . . . . . . . . . 35

\begin{tabular}{|lll}
\hline & Scale Sensitivity & 38
\end{tabular} 
$\begin{array}{lll}5 & \text { Experiment } & 40\end{array}$

5.1 Experimental validation $\ldots \ldots \ldots \ldots \ldots \ldots$

5.1 .1 Computational Cost . . . . . . . . . . . . . . . . . 40

5.1 .2 Detection Results . . . . . . . . . . . . . . . . . . . . . . 42

5.1 .3 Segmentation Results . . . . . . . . . . . . . . . . . 43

5.1 .4 Tracking Results . . . . . . . . . . . . . . . . . . . 44

5.2 Hardware in the loop simulation . . . . . . . . . . . . . . 44

6 Concluding Remarks 51

6.1 Summary . . . . . . . . . . . . . . . . . . . . . 51

\begin{tabular}{ll}
\hline References & 53
\end{tabular}

\begin{tabular}{ll}
\hline Appendices & 61
\end{tabular} 


\section{ABBREVIATIONS}

\begin{tabular}{ll}
\hline Symbol & Meaning \\
\hline CV & Chan-Vese \\
KF & Kalman Filter \\
HT & Hough Transform \\
ROI & Region of Interest \\
HIL & Hardware in the Loop \\
LSD & Line Segment Detector \\
SVM & Support Vector Machine \\
HIS & Hue Saturation Intensity \\
UAV & Unmanned Aerial Vehicle \\
GPS & Global Positioning System \\
RANSAC & Random Sample Consensus \\
PCA & Principle Component Analysis \\
GIS & Geographic Information Systems \\
ARHT & Adaptive Random Hough Transformation \\
KACST & King Abdulaziz City for Science and Technology \\
\hline
\end{tabular}

\section{LIST OF SYMBOLS}

\begin{tabular}{ll}
\hline$\nabla$ & Gradient \\
$E$ & Energy Function \\
$\Omega$ & Domain of the Image \\
$\theta$ & Angle of Line Segment
\end{tabular}




\section{LIST OF FIGURES}

1.1 Different UAV models. . . . . . . . . . . . . . . . . . . . . . . 13

1.2 Different UAV Applications. . . . . . . . . . . . . . . . . . . . . . 14

1.3 Canny edge detection results on experimental landing video image . . 16

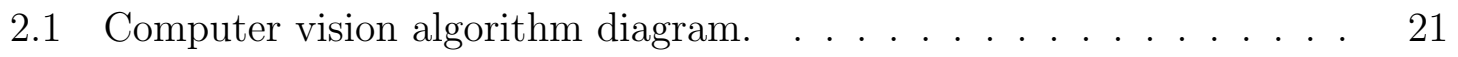

2.2 Different runway configurations. . . . . . . . . . . . . . . . . 23

2.3 Runway Model . . . . . . . . . . . . . . . . . . . . . . . . . . . . . 24

$3.1 \quad$ Edge detectors for various orientations $\ldots \ldots \ldots \ldots \ldots$

3.2 Energy profile and candidate lines . . . . . . . . . . . . . . . . . . . . 29

$3.3 \quad$ Visualization of the Kalman Filter performance in tracking . . . . . . 37

4.1 True positive rate in function of the scale . . . . . . . . . . . . . . 39

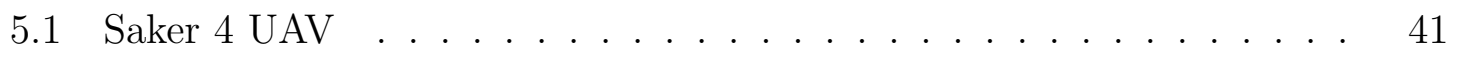

5.2 Output of the runway detection algorithm $\ldots \ldots \ldots \ldots 42$

$5.3 \quad$ GD evolution for all possible types of initialization. . . . . . . . . . 43

5.4 Hardware in the Loop simulation system . . . . . . . . . . . 45

5.5 Video sequence 1 showing the tracking output . . . . . . . . . . 46

5.6 Video sequence 2 showing the tracking output . . . . . . . . . . . 47

5.7 Video sequence Xplane 1 showing the tracking output . . . . . . . . 48

5.8 Video sequence Xplane 2 showing the tracking output . . . . . . . . . 49

5.9 Video sequence Xplane 3 showing the tracking output . . . . . . . . 50 


\section{Chapter 1}

\section{Introduction}

Quite recently, Unmanned Aerial Vehicles (UAVs) have become more and more prevalent, enabling tasks that are too dirty, too dull or too dangerous to be undertaken by manned flying vehicles.

UAVs have various civil and commercial applications. For instance, they have been used in environmental surveillance applications, including fire detection [1], event detection [2], object tracking such as vehicles [3], [4, [5], or traffic and crowd monitoring. Also, it has been used in crop management and agriculture activities including precision farming, spraying crops [6], or collecting high-spatial resolution images of the crops [7]. Moreover, oil and gas industry has used UAVs for pipeline inspection in order to maintain their value, protect them effectively against damage, and supervise the transmission process [8]. In addition to that, telecommunications sector has plans for utilizing UAVs to act as surrogate low altitude satellites and to run signal coverage surveys [9]. Structure detection is also an application, including detection of buildings [10], highways [11], and canals. Monitoring is another use for UAVs, river boundaries, bridges, coastlines [12], flash flood in desert [13], and pollution for control and air sampling purposes are targets to be monitored.

On the other hand, UAVs play an increasing role in military applications [14], such as performing aerial and maritime surveillance, engaging in battlefield management, patrolling and mapping, providing secure telecommunications [15], removing mines 
activities, guiding shells and missiles, recognition targets, or even in providing wide area munitions deployments [16]. They are also used for helping police agencies, by collecting evidences, providing assistance in hazardous conditions before compelling [17], or aiding in search and rescue operations [9]. As one can see how tremendous the field of applications is. This gives high indication about the importance of getting such technology work more efficiently and robustly.

One of the main practical challenges associated with the operation of fixed wing UAVs is to guarantee a safe takeoff and landing [18], particularly since UAVs are more sensitive to turbulence than heavier manned aircrafts. Automatic takeoff and landing is typically carried out using navigation sensors, such as absolute positioning systems (e.g. GPS), accelerometers, gyrometers and magnetometers. While the fusion between GPS and inertial unit-based measurements greatly improve positional accuracy, the residual positional errors are too large to allow reliable UAV landings on a practical UAV airstrip. Positioning accuracy on practical UAV airstrips could be improved by using enhanced GPS systems (such as differential GPS). Nevertheless, such systems require additional equipment, such as reference stations, which are costly. By contrast, the ever decreasing cost and increasing performance of cameras make them particularly suitable to this application. Since UAVs are mainly used for surveillance applications, the vast majority of UAV flights are conducted during good weather conditions, during which the visibility is high. In order to correct for the GPS positioning errors, we propose a new algorithm leveraging image processing and control systems theory to detect the runway edges, and use this information to correct the longitudinal and lateral position of the UAV during the landing phase. 

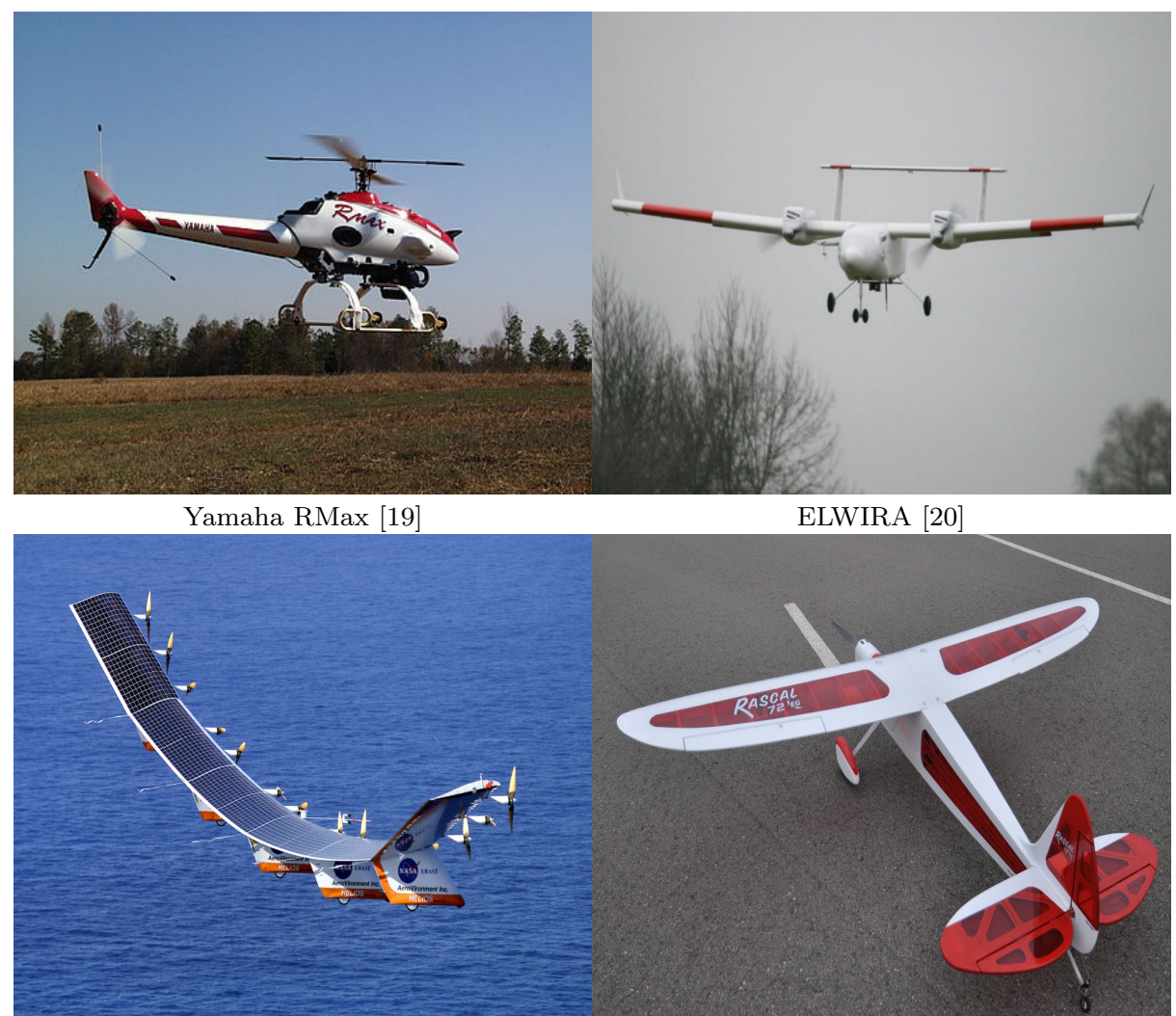

NASA Pathfinder solar powered [21]

Sig Rascal 22

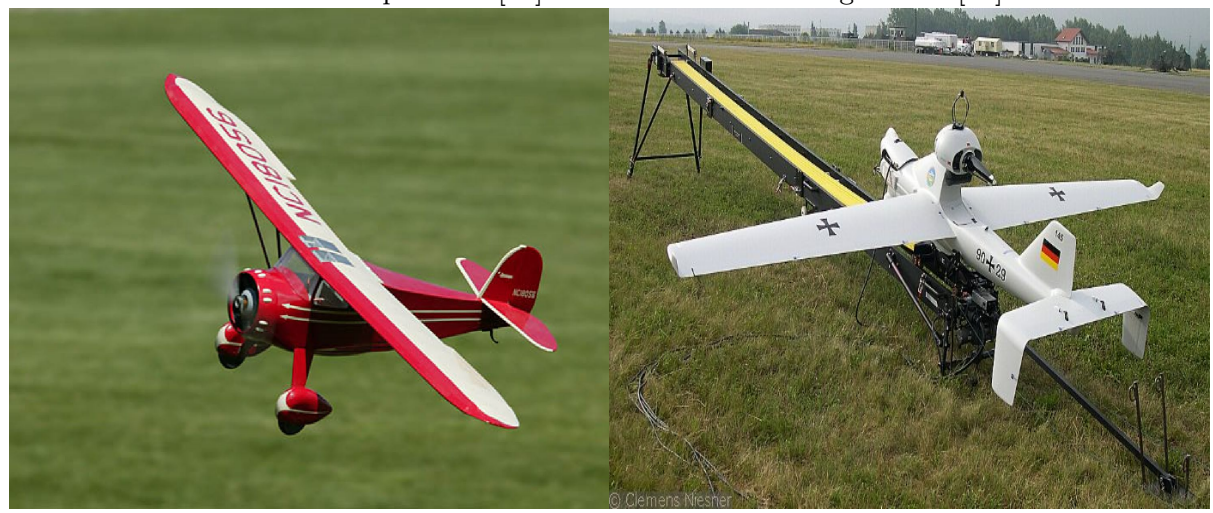

Monocoupe A90 23]

LUNA X-2000 24]

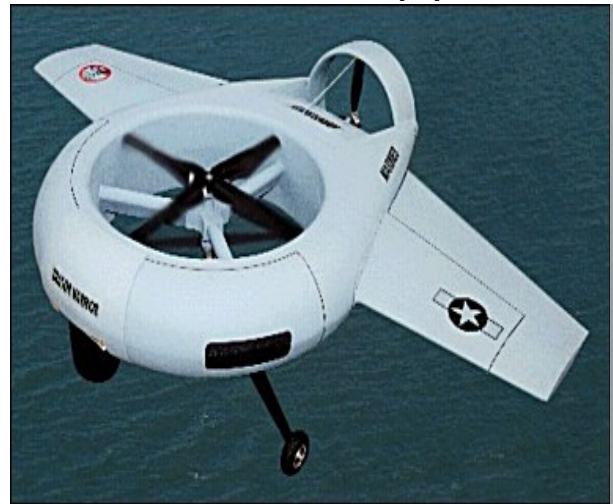

Cypher [25]

Schiebel 26]

Figure 1.1: Different UAV models. 


\section{4}

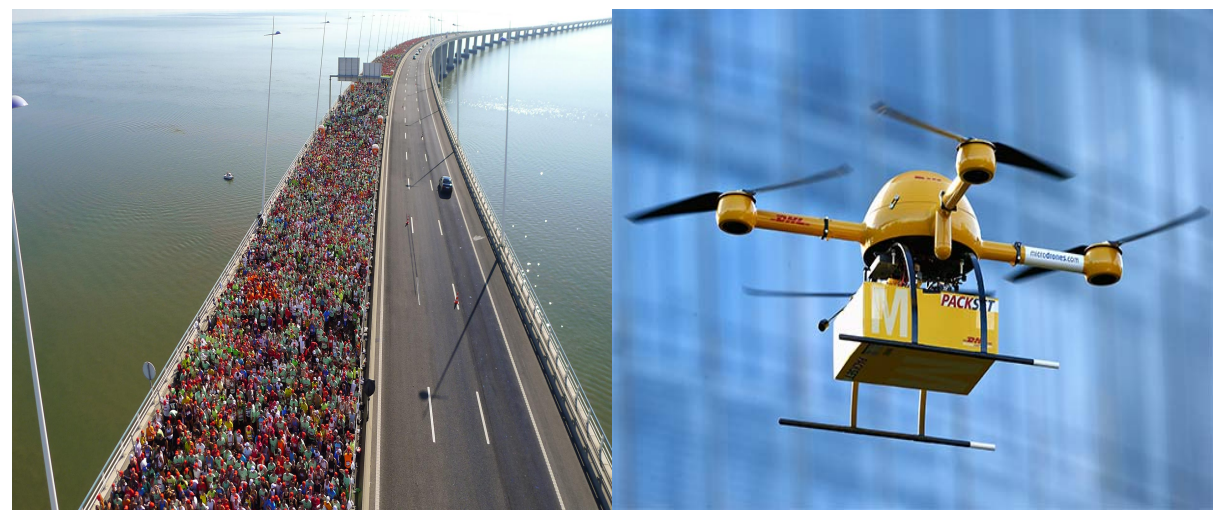

Crowd Monitoring

Package Delivery Services

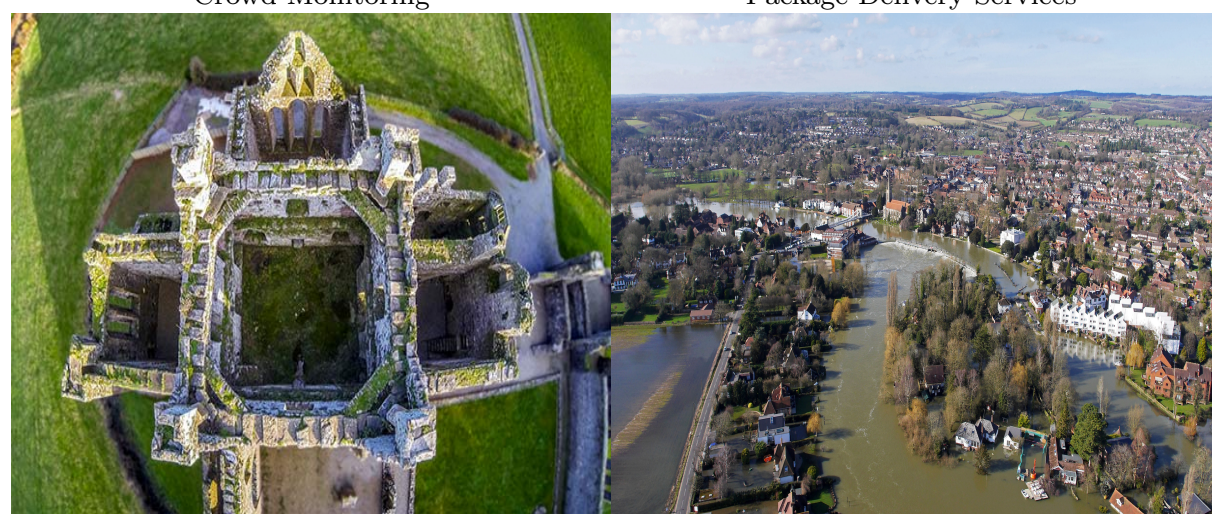

Drone Cinematographer

Flood Monitoring

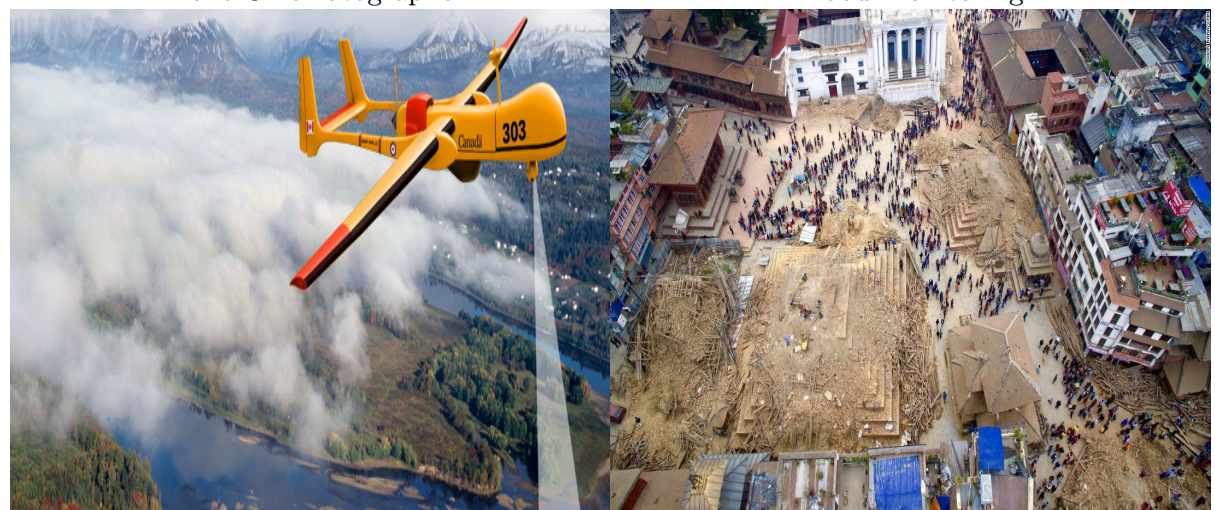

Search and Rescue

Disaster Management

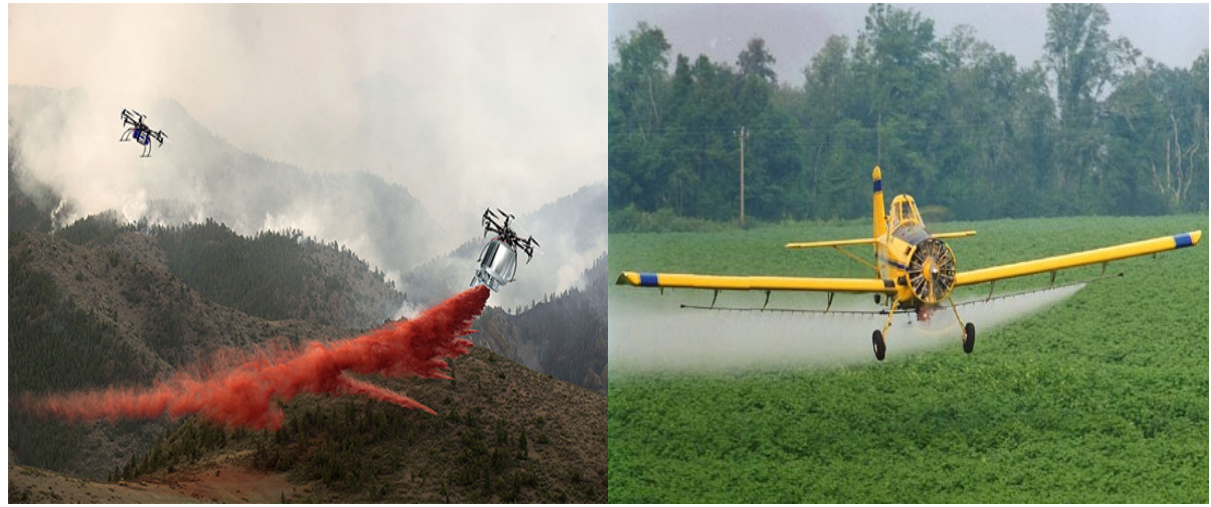

Forest Fire Control

Crop Dusting

Figure 1.2: Different UAV Applications. 


\subsection{Objectives and Contributions}

The main contribution of the proposed algorithm is to allow a very robust (under all lighting conditions or runway configurations) detection and tracking of the runway edges, to guarantee an accurate landing on narrow airstrips. It enables UAV to land on any arbitrary runway. In other words, there is no need to pre-defined geometrical configuration of the runway which requires to exactly fit the runway including taxilanes, for each time of day (illumination change) including rain, shadows, etc. While you can create a few models, and will work under pre-defined conditions, but not for the immense variability of illumination conditions. Our method avoids pre-defined models, and takes a discriminative approach whereby a runway is something straight that differs statistically from the nearby surrounding conditions. This flexible model avoids the issue of unscalability of generating appearance templates.

Our objective is to develop such a robust scheme that uses a minimal number of assumptions on the shape of the runway, robust video detection, and tracking method coupled with a KF which can fuse additional information (such as gyrometer data) in its prediction step. We illustrate this sensitivity to model parameters in Figure 1.3. which shows the difficulty of robustly selecting the contours of the image that are relevant to the proposed problem. This figure illustrates the considerable modification of the output of a Canny Edge Detector[27] (applied to a UAV landing video) due to minor changes in its parameters.

The remaining sections are organized as follows. Section 2.1 gives a high level overview of the proposed runway detection and tracking algorithm. The core visionbased feature extraction algorithm is described in section 2.2, and formulated as an optimization problem. After that, the detection and segmentation algorithms are presented in section 3.1 and section 3.2 . Section 3.3 introduces the KF formulation of the runway edge detection and tracking algorithm. In section 5.1, the performance of this algorithm is validated using experimental landing videos obtained by the Saudi 
Department of Aerospace. In this section, we show that runway detection and tracking is achieved in a very wide variety of runway configurations, lighting conditions and obstacles, without any modification of the parameters of the algorithm. To further illustrate the robustness of the proposed scheme, we develop a hardware-in-the-loop (HIL) framework around a commercial flight simulator and the proposed runway tracking algorithm in section 5.2 .

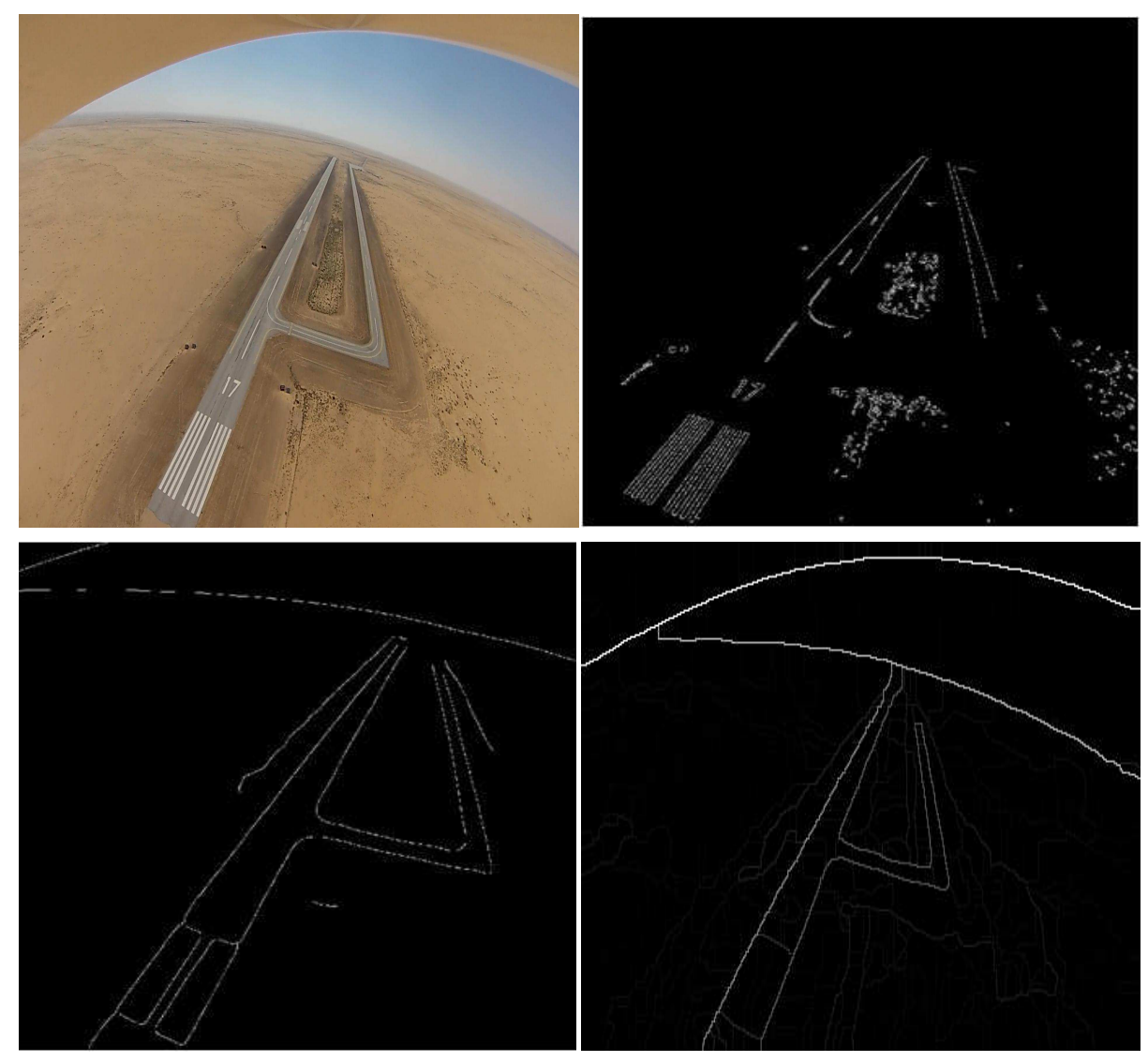

Figure 1.3: Canny edge detection results on experimental landing video image. The upper left subfigure shows the actual video data, while the upper right, lower left and lower right subfigures correspond to the outputs of the Canny Edge detector applied to this image, using the parameters $\sigma=1$ (upper right), $\sigma=10$ (lower left) and $\sigma=20$ (lower right), in which $\sigma$ denotes to the standard deviation of the Gaussian filter. All these results where fixed to a threshold [0.1-0.4]. As can one see from these pictures, a small variation of parameters dramatically affects the features that can be extracted from the image. Recent edge detectors [28] exhibit similar behavior. Output suffers from false positives and it is not real time. 


\subsection{Related Work}

Numerous computer vision algorithms have been proposed to help the navigation of fixed wing and rotary UAVs. However, to the best of the authors knowledge, no robust runway detection and tracking scheme capable of working under a variety of lighting conditions and runway configurations is currently available. Nowadays, the available methods, such as [29] require the user to specify a very large number of parameters associated with the current tracking problem in advance. Moreover, it requires using a rendered model of the runway and attempted to match it to the image which requires an appearance model (e.g., a pixel-level intensity map that describes the appearance of the runway). In addition to that, some methods need to specify which features (for instance landmarks or shadows [30], [31], [32]) have to be excluded from the tracking problem in advance. In [33], the authors use morphological image processing and Hough Transforms (HTs) to identify the horizon and estimate the attitude of a UAV. The authors of [34] proposed a template matching algorithm aided with thresholding to detect and track the position of a runway. Active methods can also be thought of, for instance in the article [35], which uses active infrared emitters to guide the UAV during its landing. In article [36], the charge coupled device imaging system parameters are used and regulated to enhances the contrast between road region and lane lines and then used an improved HT to detect the lines.

Needless to say, color features have been excessively used in such segmentation or detection schemes, for example, in [37], the road and the background are classified into two different regions according to their color features in Hue Saturation Intensity (HIS) color space, while in [38], color features are combined with texture features and then correlated with Gabor Filter or with Sobel Filter as in [39]. In [40], the authors estimated the boundaries of the road based on color features and then fit a road curvature model using vanishing points. In addition to that, in [41, an illuminantinvariant feature space is combined with a road class-likelihood classifier in order to 
identify the road pixels without using any priors on the shape of the road. Adding to that, authors of [42] used a hierarchical approach for road detection, starting from over segmentation the pixels into superpixels level based on road probability density map, throughout accumulating the neighbors of superpixles into two different regions using GrowCut. Also, it is stated in [43] that a classifier is trained over RGB color space and used parallel computing and hierarchical mapping of the 2D image to make it fast. Nevertheless, if geographic information systems (GISs) dataset is provided, it can be combined with road cues and then segment the road [44].

In other contexts, computer vision has been successfully used to detect road lanes (for autonomous vehicle applications), for instance in [45] where roads are detected using HT and Robert Filter. The authors of [30], use splines curve fitting, HT, Canny Edge Detector, and vanishing points to estimate the boundary of the road. While effective, this method is complex and does not run in real time on commercially available embedded platforms. In article [46] a Line Segment Detector (LSD) is used to detect the main line features, and K-means clustering is applied to sort and select specific lines. The authors of [47] have a different approach, relying on a Sobel Filter and K-means to find the edges of the road from a fixed surveillance camera, while in [48], Principle Component Analysis (PCA) is used with K-means are used to fit smooth splines and detect the lane. In [49], [50], the lane markings and lane texture and the vehicle state information are incorporated with Steerable filters to have lane marking detection. The authors in [51] relied on estimating the positions of the road lanes based on the gradient of the image in multiple regions of interest and fusing the measurements assuming smooth and continuous change in the location of the road. In contrast to that, in [52], the authors introduced the concept of ridgeness instead of the gradient where its output is more reliable.

Other approaches for lane or road detection exist, such as in [53], where Random Sample Consensus (RANSAC) and cubic spline curve fitting are used to extract the 
lane position from a video stream. A linear parabolic lane tracking system with KF is proposed in [54], while the authors of [55] proposed edge distribution functions along with modified HT for road detection, and fitted a linear parabolic model to track the road. Moreover, Support Vector Machine (SVM) pattern recognition has been used to extract road surface [56], [57]. However, authors of [58] favored artificial neural networks over SVM to classify the pixels of the image and then combined cubic-spline curves fitting with RANSAC to achieve the detection step, and followed that with Particle filtering step to achieve tracking step.

This filter is widely used for tracking, though it could not be fast enough for real time applications, for example but not limited to, authors of [59] used it after they applied Tabu Search algorithm and Adaptive Random Hough Transformation (ARHT) for lane detection. Also, it has been used in [60], after using Canny Edge Detector to find the lane marking followed by a confidence measure method to validate the candidate markings. On the other hand, many approaches, such as [61] tend to use convex methods in PDE optimization to segment the image into $N$ regions based on global intensity statistics or local intensity statistics. 


\section{Chapter 2}

\section{Algorithms Overview and Runway Mathematical Model}

\section{Representation}

In this chapter, the high level overview of the runway edge detection and tracking algorithms are presented. Next, a model for aerial images containing a runway is formulated. Then, it is used to formulate an optimization problem to detect and segment the runway given an image. After that, optimization methods for the energy are derived which will be used in the next sections for the detection and segmentation steps.

\subsection{Runway detection and tracking algorithms overview and structure}

The runway edge detection and tracking algorithms are based on the combination of computer vision and classical Kalman Filtering. First of all, the streaming video data is converted into a sequence of frames, which are downsampled (both in the color space and spatially). The regions of interest (ROI) of the image (containing 
the runway) is detected at this step. Once the ROI is identified, the edges of the runway are detected through computer vision techniques detailed in section 3 . The edge location estimates are then fed to a $\mathrm{KF}$, which can fuse additional data (if available) to estimate the runway location. Then runway edges are displayed on the real-time video, or can be used to feed the UAV autopilot (the location of the runway with respect to the UAV can be computed modulo a conversion of the video image coordinates to absolute coordinates).

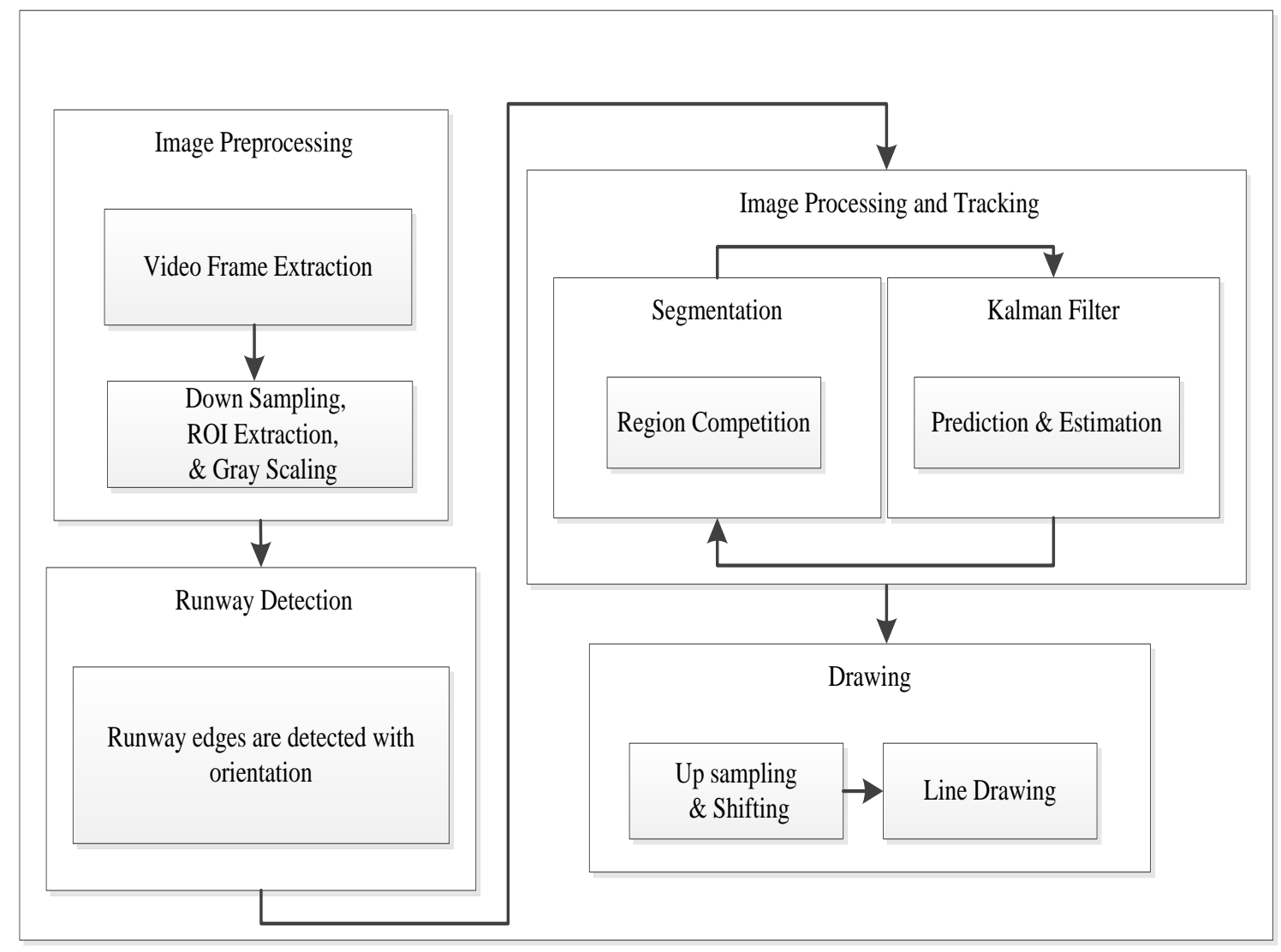

Figure 2.1: Computer vision algorithm diagram. 


\subsection{Generic Model Representation and Energy Op- timization}

Our key innovations are a model and optimization methods that are specifically designed to lead to efficient computational algorithms that can be implemented on embedded platforms on off-the-shelf UAVs. Further, the algorithms are designed to be robust to background clutter, illumination conditions (e.g., day and night), and noise - all important visual nuisances that are prevalent in aerial imagery.

Based on the nature of the runways that UAV lands on, our model for a runway is two line segments, one for each edge of the runway. Typical runways are flat enough to be approximated as two parallel straight lines, which due to perspective projection may appear non-parallel in the image. Although runways may consist of multiple intersecting runways, in which case the two line segment assumption may fail, we wish to detect the runway localized to a certain region below the aircraft, where the two lane segment assumption is largely true. See Figure 2.2, The advantage of such a model is that this runway model is finite dimensional and leads to fast algorithms. Our model of the image consists of two segments (the runway), and that for each line segment, a neighborhood region on each side of the segment consists of maximally different image intensity statistics. See Figure 2.3. No assumptions are assumed on the rest of the image (e.g., the background and the interior of the runway) as the images may vary significantly in this region depending on the environment.

For simplicity, it is assumed that the aircraft is approximately aligned to the runway, which is true in our application. This assumption holds since the navigation system based on GPS is able to position the aircraft within a few meters (typically less than 15 meters), and a heading uncertainty of a few degrees (typically less than 10 degrees). In this case, within a rectangle around the runway, we may assume that each line segment (representing the runway) intersects the top and bottom of 

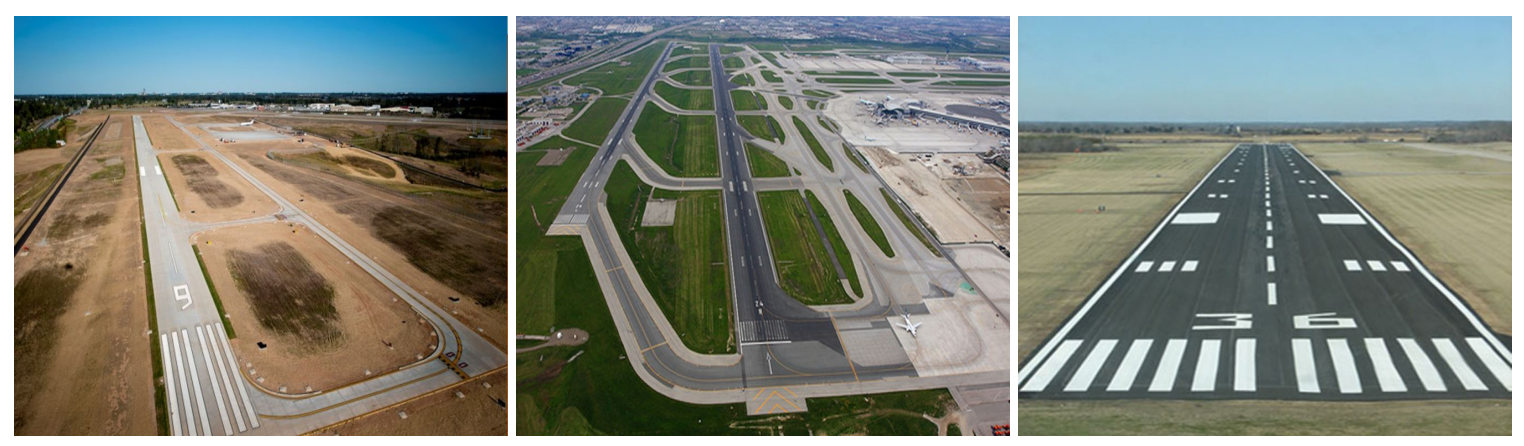

Figure 2.2: Different runway configurations. It is common to have different statistics inside the runway than the background in the surrounding area.

the rectangle. Thus, the approximate localization of the runway within the image is represented by this rectangle. Therefore, the line segments may be specified by four distances $d_{i}, i=1, \ldots, 4$ between the corners of the rectangle and the endpoints of the line segments. See Figure 2.3 .

We now formulate an optimization problem defined on line segments (specified by $\left.d_{i}\right)$ such that the minimizer corresponds to the line segments that are aligned with the edges of the runway. As noted earlier, the runway is such that the neighboring regions on either side of the line segments have maximally differing image intensity statistics. For simplicity, we assume that the statistics is the mean RGB values with the neighborhoods, but any other statistics may be used (e.g., intensity histograms, statistics of filter bank responses, etc). Let the image be denoted by $I: \Omega \rightarrow \mathbb{R}^{k}$ ( $k=3$ for RGB) where $\Omega \subset \mathbb{R}^{2}$, and let $R, R^{c} \subset \Omega$ denote the inside and outside neighborhood regions of the line segments, respectively. See Figure 2.3. The mean statistics $u, v \in \mathbb{R}^{k}$ for the inside and outside neighborhoods are then

$$
u=\frac{1}{|R|} \int_{R} I(x) d A, \quad v=\frac{1}{\left|R^{c}\right|} \int_{R^{c}} I(x) d A,
$$

where $|R|$ denotes the area of $R$, and $d A$ is the area element. Since we would like the line segments positioned so that $u$ and $v$ are maximally different, we define the 


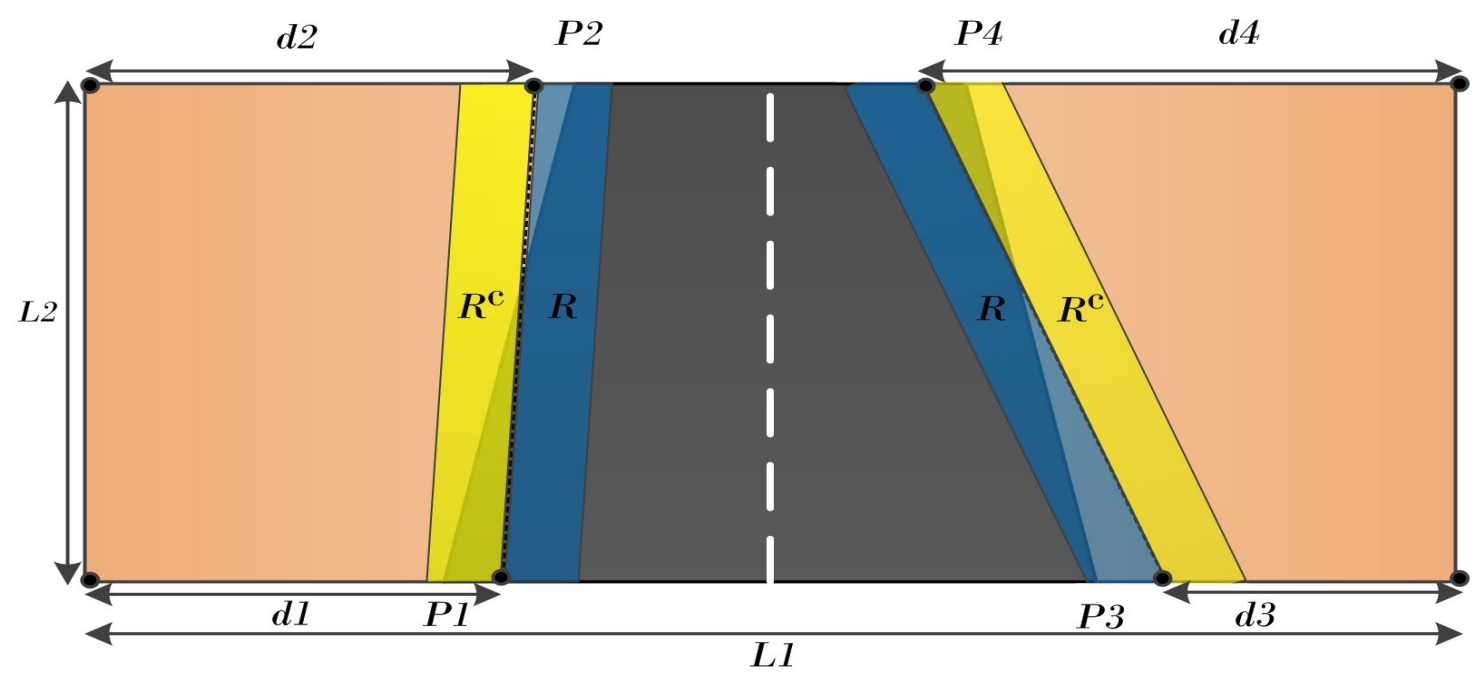

Figure 2.3: Runway Model. The runway edges are straight line segments such that the corresponding regions $R$ and $R_{c}$ have maximally different image statistics. The other labeled quantities are lengths used through the manuscript.

following energy to be minimized:

$$
E\left(d_{1}, d_{2}, d_{3}, d_{4}\right)=-\frac{1}{2}|u-v|^{2}+\gamma \sum_{i=1}^{4}\left|d_{i}-d_{i}^{\prime}\right|^{2} .
$$

Note that $u$ and $v$ are functions of the position of the line segments since $R$ and $R^{c}$ depend on the line segments, which are in turn specified by $d_{i}$. Therefore, the energy $E$ is a function of $d_{i}$.

Moreover, we added a regularization term in order to penalize over the final location of $d_{i}$ away from its initial one, denoted by $d_{i}^{\prime}$. This would prevent over segmentation in case the statistics of a region include outliers. Higher value of $\gamma$ will limit the freedom of the lines displacement toward convergence and vise versa.

As we will see, the use of region statistics in the design of the energy leads to a robust runway detection algorithm. Local derivatives [45], which are represented by edge detection filters widely used in edge detection, are sensitive to noise and extraneous features in the images. Thus, using statistics that are more robust to such disturbances will lead to an algorithm that is less sensitive to irrelevant image 
features. The larger the neighborhoods, $R$ and $R^{c}$, the more robust the statistics are to local disturbances. However, larger neighborhoods encompass more of the background where our assumptions of maximally differing statistics of $u$ and $v$ may not hold due to irrelevant clutter. This tradeoff in size is important and will be analyzed in the subsequent sections.

The energy above is related to a large body of literature in image segmentation by partial differential equations based methods [62, 63, 64] in which the energies are defined on the set of (infinite dimensional) regions. The energy is most closely related to [65], where the objective is to divide the image into two disjoint regions that have maximally different intensity means. Our energy formulation has two major modifications with respect to previously used formulations: first, the energy is defined on a simplified representation of regions defined by line segments to support real-time applications needed for the UAV, and second, the use of neighborhoods rather than the entire image to avoid background clutter typical in aerial imagery. One problem with the methods of image segmentation using partial differential equations is they typically rely on local descent methods because the energies are non-convex (as is our energy of interest). Therefore, they require the user to design an initialization algorithm. Our model of the runway is designed to achieve a computationally tractable real-time detection algorithm that localizes the runway. This supports a fully automatic real-time runway detection and segmentation algorithm, a key advance with respect to existing image segmentation methods, and the key computer vision innovation in our method.

In the next subsections, an automatic detection algorithm that roughly localizes the runway by approximating an optimizer of $E$ is described, and then an optimization scheme that refines the detection to achieve a more accurate localization of the runway is presented. 


\section{Chapter 3}

\section{Image Processing and Tracking}

\section{Algorithms}

In this chapter, a fast and robust algorithm to detect the runway and find its location is designed. Next, the energy function is optimized in order to find the exact location of the runway lines in each frame using gradient decent method. Finally, KF with the segmentation algorithm are integrated to have robust tracking algorithm.

\subsection{Detection Method}

The optimization of $E$ directly by evaluating $E$ for every $d_{i}$ (or a sampling of the space) would be computationally prohibitive, certainly prohibitive for real-time applications. Moreover, without an initialization near the desired runway, local optimization techniques, which are the only candidates for optimization of $E$ due to non-convexity, would fail to capture the runway. Therefore, in this section, a coarseto-fine algorithm is constructed such that it searches efficiently over the space of $d_{i}$. The algorithm prunes away irrelevant line segments in the search space of $d_{i}$ and proposes a few candidate segments as segments corresponding to the runway. The energy

of these few candidates can then be evaluated, and the energetically most favorable candidate can be selected. This leads to a real-time fully automatic algorithm that 
is also robust to clutter.

First, to efficiently propose candidate pairs of line segments, we decouple the pair and focus on generating single line segments as candidates. Pairs of line segments corresponding to candidates of the runway will be proposed from the single line segment candidates and will be described later in this subsection. Thus, our algorithm initially searches for candidate line segments representing any edge of the runway. This is accomplished by representing a line segment by an angle $\theta(\theta \in[-\pi / 2, \pi / 2]$, which corresponds to the angle of the line segment with respect to the $y$-axis, and a location $x$ ( $x$-coordinate of the center of the line segment). Our algorithm efficiently computes minimizers of

$$
E^{\prime}(x, \theta)=-\frac{1}{2}\left(u^{\prime}(x, \theta)-v^{\prime}(x, \theta)\right)^{2}
$$

where $u^{\prime}$ and $v^{\prime}$ are the mean values of the image in the neighborhoods on either side of the line segment specified by $x, \theta$.

Evaluating $E^{\prime}$ for all $x, \theta$ is still too computationally prohibitive, and thus we introduce a hierarchical coarse-to-fine search. Instead of computing $u^{\prime}, v^{\prime}$ for each $\theta$, i.e., the mean intensity on either side of a slanted line segment, we design a scheme whereby the energy $E^{\prime}$ is low provided there is some $\theta \in[-\pi / 2, \pi / 2)$ for which $E^{\prime}$ is low, and only a single computation of means is required (rather than computation of means for all $\theta$ ). A diagram illustrating the neighborhoods (called $r_{+}, r_{-}$) of the line segment, in which $u^{\prime}, v^{\prime}$ are computed, for various $\theta$ is shown in Figure 3.1. Consider the intersection of the neighborhoods $r_{+}$and $r_{-}$for each $\theta$ between some range of angles $|\theta|<\pi / 2$. The intersected regions are shown in the bottom right in Figure 3.1. Note that $E^{\prime}$ is low for some angle $\theta$ when the difference of means in the intersections of $r_{+}$and the intersections of $r_{-}$is large. We call the difference of means in the intersected regions squared the response $\mathcal{R}$, which is a function of the location $x$. 


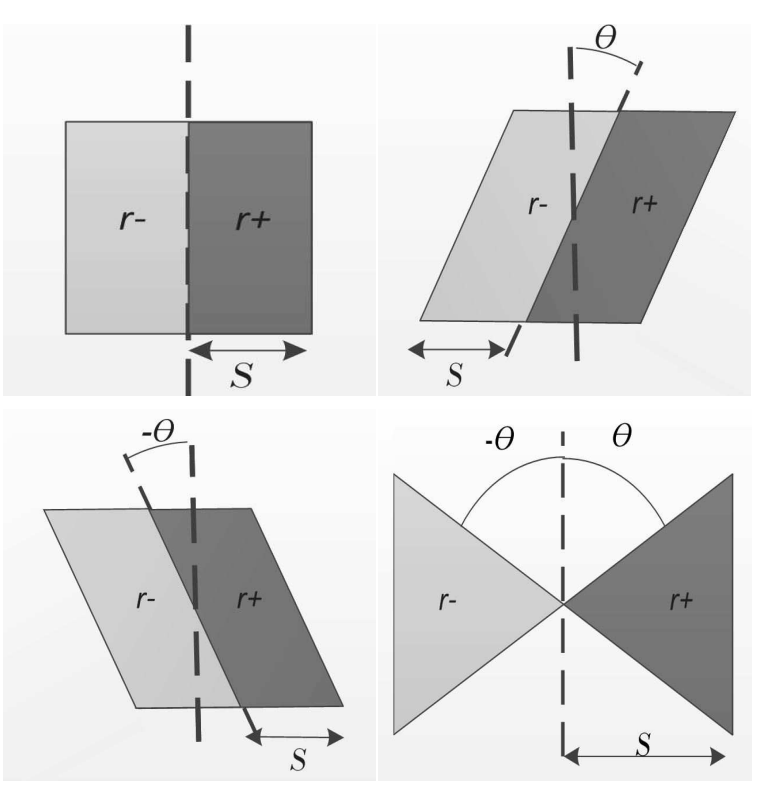

Figure 3.1: Edge detectors for various orientations. The lower right regions is the intersection of all $r+$ and $r_{-}$within a specified range $\left[-\theta_{m}, \theta_{m}\right]$; it is able to detect lines with any orientation without searching within the range.

The candidate locations of line segments corresponding to edges of the runway are local maxima of $\mathcal{R}$ with the highest responses. Note that since the intersection of regions $r_{+}$has fewer pixels than any particular $r_{+}$for a fixed angle, the means are more susceptible to noise, and therefore, computing local minima may generate false positives. However, these false positives are pruned out in the next step. Clearly, false positives are the cost for efficiency, i.e., avoiding the search over all $\theta$ at all locations. Since locating local maxima of $\mathcal{R}$ gives only candidate locations, the energy $E^{\prime}$ is computed at multiple $\theta$ (we choose $\theta \in\{-\pi / 4,-\pi / 8,0, \pi / 8, \pi / 4\}$ ) only at the local maxima locations. This gives approximations for the orientation of the line segments. We have thus avoided an expensive search over two variables $(x, \theta)$ and only a search over $x$ to find local minima of $\mathcal{R}$ is needed with this procedure. Fortunately, computation $\mathcal{R}$ for all $x$ is efficient enough to be implemented in real-time. This procedure gives candidates for line segments of the runway, $\left(x_{i}, \theta_{i}\right), i=1, \ldots, N$ where $N$ is chosen small (in our experiments, $N=5$ ). This is illustrated in Figure 3.2 .

We now compute the energy $E$ for pairs of line segments in the candidate list 

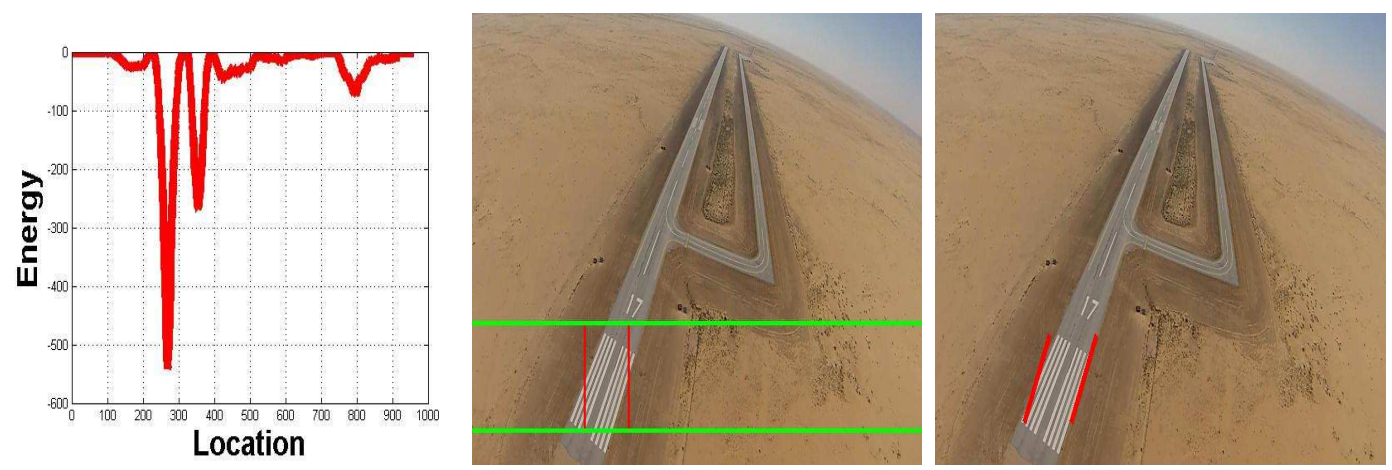

Figure 3.2: Energy profile and candidate lines. Left: Energy profile for the image between the green lines. Middle: Red lines are chosen among the candidate edges based on the correlation matrix. Right: Red lines are edges with correct orientation.

$\left(x_{i}, \theta_{i}\right)$. The pair with the lowest energy is selected as the detected runway. The energy $E$ is thus evaluated $N^{2}$ times. Since $N$ is small, this is a considerable time savings compared with evaluating $E$ at all $d_{i}$. In fact, we demonstrate in the experiments that our entire detection method is real-time. The right image in Figure 3.2 shows an example result of the final minimum energy line segment pair.

The algorithm for this detection procedure is summarized in Algorithm 1.

1. for each $x \in\left\{0,1, \ldots, L_{1}-1\right\}$

- compute $u$ and $v$ with regions defined by the mask in Fig. 3.1 (bottom, right) and centered at $\left(x, L_{2} / 2\right)$ for $\theta=\pi / 4$

- compute $\mathcal{R}(x)=-(u-v)^{2} / 2$

2. compute the $N$ lowest local minima of $\mathcal{R}$, called $x_{i}, i=1, \ldots, N$

3. compute the $\theta \in\{-\pi / 4,-\pi / 8,0, \pi / 8, \pi / 4\}$ corresponding to $x_{i}$ that minimizes $E$ for $i=1, \ldots, N$

4. compute $E$ for all pairs of line segments determined by $\left(x_{i}, \theta_{i}\right)$

5. output the pair of line segments $\left(x_{i}, y_{i}\right),\left(x_{j}, y_{j}\right)$ that have minimum $E$ 


\subsection{Local Optimization for Refined Segmentation}

Now, we derive a local optimization method for $E$ given an initialization close to the desired runway. This step is needed for two reasons. First, the detection procedure in the previous section only gives a coarse approximation of the runway, which needs to be refined. Second, the algorithm will be used to track the runway in a video, and thus the lane is already roughly localized (using the result from the previous frame), and the line segments only need to be slightly refined. Local optimization is computationally fast, and less costly than running the global detection procedure described in the previous section. Therefore, a gradient descent method is presented to optimize $E$ [66], [67]. One may interpret the energy $E$ as defined on a closed contour, i.e., a trapezoid formed by the line segments. The advantage of this interpretation is that, we may use results in the PDE image segmentation literature [62, 63, 64], which have derived methods for optimizing arbitrary energies defined on closed contours that are allowed to deform in arbitrary ways that may change shape. However, in optimizing $E$, we would like to enforce the constraint that the trapezoid remains a trapezoid. Fortunately, such a constraint can be enforced by restricting the deformations of the contour to trapezoid preserving deformations [68].

The computation of the gradient of $E$, considering the fitting term, $\nabla E=\left(\partial E / \partial d_{i}\right)_{i=1}^{4}$, is summarized in the proposition next. One can see that each partial derivative in the gradient can be efficiently computed by a line integral of the line segments.

On the other hand, the gradient of the regularization term is given by $2 \gamma\left(d_{i}-d_{i}^{\prime}\right)$, and it will be added to the one corresponding to fitting term of the energy function derived in the preposition.

Hence, he gradient descent algorithm to minimize this energy is then given by

$$
\vec{d}_{k+1}=\vec{d}_{k}-\Delta t \nabla E\left(\vec{d}_{k}\right),
$$


where $\vec{d}_{k}$ is the vector of $d_{i}$ 's at each iteration of the gradient descent, and $\Delta t=$ $0.5 /\left|\nabla E\left(\vec{d}_{k}\right)\right|$ is the time step size. Note that $\vec{d}_{0}$, the initialization, is the result of the detection described in the previous section at the beginning of the video. For subsequent frames, it is the prediction from the previous frame (see next section for details).

Proposition 1. The gradient of $E$ is given by $\nabla E=\left(\partial E / \partial d_{i}\right)_{i=1}^{4}$ where the partial derivatives are given by:

$$
\begin{aligned}
\frac{\partial E}{\partial d_{1}}=L_{2}(v-u) & {\left[\frac{1}{|R|} \int_{0}^{1}(t-1)\left(I\left(C_{L_{1}}(t)\right)-u\right) d t\right.} \\
+ & \left.\frac{1}{\left|R^{c}\right|} \int_{0}^{1}(t-1)\left(I\left(C_{L_{1}}(t)\right)-v\right) d t\right] \\
\frac{\partial E}{\partial d_{2}}=L_{2}(v-u) & {\left[\frac{1}{|R|} \int_{0}^{1} t\left(I\left(C_{L_{1}}(t)\right)-u\right) d t\right.} \\
+ & \left.\frac{1}{\left|R^{c}\right|} \int_{0}^{1} t\left(I\left(C_{L_{1}}(t)\right)-v\right) d t\right], \\
\frac{\partial E}{\partial d_{3}}=L_{2}(v-u) & {\left[\frac{1}{|R|} \int_{0}^{1}(t-1)\left(I\left(C_{L_{2}}(t)\right)-u\right) d t\right.} \\
+ & \left.\frac{1}{\left|R^{c}\right|} \int_{0}^{1}(t-1) I\left(\left(C_{L_{2}}(t)\right)-v\right) d t\right], \\
\frac{\partial E}{\partial d_{4}}=L_{2}(v-u) & {\left[\frac{1}{|R|} \int_{0}^{1} t\left(I\left(C_{L_{2}}(t)\right)-u\right) d t\right.} \\
+ & \left.\frac{1}{\left|R^{c}\right|} \int_{0}^{1} t\left(I\left(C_{L_{2}}(t)\right)-v\right) d t\right],
\end{aligned}
$$

where $C_{L_{i}}$ is the arc-parameterization of the $i^{\text {th }}$ line segment corresponding to $\left(d_{1}, \ldots, d_{4}\right)$, $L_{2}$ is the image width, and $u$ and $v$ are the means inside the regions $R$ and $R^{c}$, respectively.

In the following subsection, the derivation of those expressions are explained in more detailed scheme. 


\subsubsection{Computation of the Gradient of $E$}

In this subsection, we show the details for the computation of the gradient of $E$

with respect to $\vec{d}=\left(d_{1}, d_{2}, d_{3}, d_{4}\right)$, considering the fitting term of the energy function. Using the Chain Rule, we see that

$$
\nabla E(\vec{d})=-(u(\vec{d})-v(\vec{d}))(\nabla u(\vec{d})-\nabla v(\vec{d}))
$$

where $\nabla$ deontes the gradient with respect to $\vec{d}$.

It remains to compute the gradient of $u$ and $v$ with respect to $\vec{d}$, which are defined by

$$
u(\vec{d})=\frac{\int_{R} I(x) d A}{\int_{R} d A}, \quad v(\vec{d})=\frac{\int_{R^{c}} I(x) d A}{\int_{R^{c}} d A},
$$

where $R$ is the banded region inside the trapezoid determined by $\vec{d}, R^{c}$ is the banded region outside the trapezoid determined by $\vec{d}$, and $d A$ is the area differential element. To compute the gradient, we may use the result [69] which states that the directional derivative of a functional

$$
e(R)=\int_{R} f(x) \mathrm{d} A
$$

is given by

$$
d e(R) \cdot h=\int_{\partial R} f(x) \mathrm{d} s
$$

where $h$ is a perturbation of the boundary of $R, \partial R$ is the boundary of $R$ that is allowed to vary, $d s$ is the arclength differential element, and $d e(R) \cdot h$ is the change in $e$ by perturbing $R$ by a perturbation $h$ (defined on the boundary of $\partial R$ ).

By applying the Quotient Rule and the previous result, one can show that

$$
\begin{array}{r}
\frac{\partial u}{\partial d_{j}}=\frac{1}{|R|} \int_{C_{L_{i}}}(I-u) h_{j} \cdot N d s \\
\frac{\partial v}{\partial d_{j}}=-\frac{1}{\left|R^{c}\right|} \int_{C_{L_{i}}}(I-v) h_{j} \cdot N d s
\end{array}
$$


where $C_{L}:[0,1] \rightarrow R^{2}$ is the line segment corresponding to either side (right or left) of the trapezoid, $N$ is the unit outward normal to $C_{L}$, and $h_{j}$ corresponds to the perturbation of $C_{L_{j}}$ when the $j^{\text {th }}$ vertex is perturbed.

$i=1,2$, and $i=3,4$ correspond to line segments at the left side and the right side, respectively. While $j=1,2,3,4$ correspond to the four variables that should be updated. Note that

$$
C_{L_{i}}(t)=\left\{\begin{array}{rrr}
\left(\begin{array}{c}
d_{1} \\
0
\end{array}\right)+\left(\begin{array}{c}
d_{2}-d_{1} \\
L_{2}
\end{array}\right) t & \text { if } & i=1,2 \\
\left(\begin{array}{c}
L_{1}-d_{3} \\
0
\end{array}\right)+\left(\begin{array}{c}
d_{3}-d_{4} \\
L_{2}
\end{array}\right) t & \text { if } & i=3,4
\end{array}\right.
$$

and

$$
h_{j}=\frac{\partial C(t)}{\partial d_{j}}=\left\{\begin{array}{ccc}
\left(\begin{array}{c}
1-t \\
0
\end{array}\right) & \text { if } & j=1,3 \\
\left(\begin{array}{l}
t \\
0
\end{array}\right) & \text { if } & j=1,4
\end{array} .\right.
$$

Since $N$ is the outward normal to $C_{L_{j}}$, it can be related to the tangent $T$ to $C_{L_{j}}$ by $N=J T$, where $J$

$$
J=\left[\begin{array}{cc}
0 & -1 \\
1 & 0
\end{array}\right]
$$

is the rotation matrix with $\theta=-90^{\circ}$ counter clockwise. Note also that

$$
T(t)=\frac{C_{L_{j}}^{\prime}(t)}{\left|C_{L_{j}}^{\prime}(t)\right|}
$$

where $\left|C_{L_{j}}^{\prime}(t)\right|$ is the speed of the point, $s$ denotes the arc length parameter,

$$
d s=\left|C_{L_{j}}^{\prime}(t)\right| d t
$$

and $L_{j}$ will denote the length of $C_{L_{j}}$. Combining equations 3.10, 3.11, 3.13) and 
(3.14) results in

$$
N(t) . h(t) d s=L_{2}(t-1) d t .
$$

Then, the gradient direction at vertex $j=3$, for example, can be derived as follows

$$
\begin{array}{r}
\frac{\partial E}{\partial d_{3}}=-L_{2}(u-v)\left[\frac{1}{|R|} \int_{0}^{1}(t-1)\left(I\left(C_{L_{2}}(t)\right)-u\right) d t+\right. \\
\left.\frac{1}{\left|R^{c}\right|} \int_{0}^{1}(t-1) I\left(\left(C_{L_{2}}(t)\right)-v\right) d t\right]
\end{array}
$$

The same argument applies to the other $d_{i}$, and thus

$$
\begin{aligned}
\frac{\partial E}{\partial d_{1}}=L_{2}(v-u) & {\left[\frac{1}{|R|} \int_{0}^{1}(t-1)\left(I\left(C_{L_{1}}(t)\right)-u\right) d t\right.} \\
+ & \left.\frac{1}{\left|R^{c}\right|} \int_{0}^{1}(t-1)\left(I\left(C_{L_{1}}(t)\right)-v\right) d t\right] \\
\frac{\partial E}{\partial d_{2}}=L_{2}(v-u) & {\left[\frac{1}{|R|} \int_{0}^{1} t\left(I\left(C_{L_{1}}(t)\right)-u\right) d t\right.} \\
+ & \left.\frac{1}{\left|R^{c}\right|} \int_{0}^{1} t\left(I\left(C_{L_{1}}(t)\right)-v\right) d t\right], \\
\frac{\partial E}{\partial d_{3}}=L_{2}(v-u) & {\left[\frac{1}{|R|} \int_{0}^{1}(t-1)\left(I\left(C_{L_{2}}(t)\right)-u\right) d t\right.} \\
+ & \left.\frac{1}{\left|R^{c}\right|} \int_{0}^{1}(t-1) I\left(\left(C_{L_{2}}(t)\right)-v\right) d t\right], \\
\frac{\partial E}{\partial d_{4}}=L_{2}(v-u) & {\left[\frac{1}{|R|} \int_{0}^{1} t\left(I\left(C_{L_{2}}(t)\right)-u\right) d t\right.} \\
+ & \left.\frac{1}{\left|R^{c}\right|} \int_{0}^{1} t\left(I\left(C_{L_{2}}(t)\right)-v\right) d t\right] .
\end{aligned}
$$




\subsection{Tracking}

KF [70] is used to predict and the initial location of the lines before they are processed by the gradient descent, and then to arrive at a final estimate. This allows us to process fewer frames, since we may predict ahead a few frames, and increase the computational speed of the runway detection algorithm, to enable this process to run in real time. Moreover, $\mathrm{KF}$ increases the robustness against clutter and outliers that are in the field of view of the camera. Finally, it can also be used to fuse inertial measurement data (such as rotation rates measured by the onboard gyrometers) to enable video-inertial data fusion.

We assume a constant velocity plus noise model, which gives a first order approximation to the dynamics of the line segments seen through the camera. We define the state space $X_{k}$ to include the vector $\vec{d}_{k}$ and their velocities, which makes $X_{k}$ an 8-dimensional vector. We assume the measurement $y_{k} \in \mathbb{R}^{4}$ is a noisy measure-

ment of the first components of $X_{k}\left(\vec{d}_{k}\right)$, and these measurements will be obtained by the converged result of the gradient descent, described in the previous section, with initialization the predicted relevant part of the state.

The dynamical system of interest can be defined as follows:

$$
\begin{aligned}
X_{k} & =\left(\vec{d}_{k}^{T}, \vec{v}_{k}^{T}\right)^{T} \in \mathbb{R}^{8} \\
X_{k+1} & =A X_{k}+B u_{k}+\zeta_{k} \\
y_{k+1} & =C X_{k}+\eta_{k} \\
\zeta_{k} & \sim \mathcal{N}(0, Q) \\
\eta_{k} & \sim \mathcal{N}(0, U)
\end{aligned}
$$

where $\vec{v}_{k}$ is the velocity corresponding to the horizontal distances $\vec{d}_{k}, S$ is the measurement noise covariance matrix, and $Q$ the process (or model) noise covariance matrix. 
In our specific problem (constant velocity plus noise), these matrices are defined as follows 난

$$
\begin{aligned}
& A=\left[\begin{array}{cc}
\mathrm{id}_{4 \times 4} & \mathrm{id}_{4 \times 4} \\
0_{4 \times 4} & \mathrm{id}_{4 \times 4}
\end{array}\right], B=0, C=\left[\begin{array}{ll}
\mathrm{id}_{4 \times 4} & 0_{4 \times 4}
\end{array}\right] \\
& U=\left[0.01 \times \mathrm{id}_{4 \times 4}\right], Q=\left[\begin{array}{cc}
0_{4 \times 4} & 0_{4 \times 4} \\
0_{4 \times 4} & 0.01 \times \mathrm{id}_{4 \times 4}
\end{array}\right] \text {, }
\end{aligned}
$$

where $i_{4 \times 4}$ denotes the $4 \times 4$ identity matrix. Note that $X_{0}$ is chosen to be the output of the detection procedure, and zero velocity.

The KF involves two steps: prediction and estimation. The "hat" notation is used to denote predicted and estimated quantities.

1. Prediction:

$$
\hat{X}_{k \mid k-1}=A \hat{X}_{k-1 \mid k-1},
$$

and the predicted estimated covariance:

$$
P_{k \mid k-1}=A P_{k-1 \mid k-1} A^{T}+Q
$$

2. Estimation:

- Measurement error/innovation covariance:

$$
S=H P H^{T}+U
$$

- Optimal Kalman Gain:

$$
K=P H^{T} S^{-1}
$$

\footnotetext{
${ }^{1}$ In this section, we do not assume to have any available inertial or positional measurements. Such measurements could be used to modify the prediction step of the Kalman Filter (for instance the rotation rates could be used in the prediction step to recompute the predicted runway edge locations, enabling a more accurate and robust tracking).
} 
- Updated state estimate:

$$
\hat{X}_{k \mid k}=\hat{X}_{k \mid k-1}+K\left(y_{k}-C \hat{X}_{k \mid k-1}\right)
$$

where $y_{k}$ is obtained by running gradient descent (3.2) with initialization the first four components of $\hat{X}_{k \mid k-1}$.

- Updated estimate covariance:

$$
P_{k \mid k}=P_{k \mid k-1}-K H P_{k \mid k-1}
$$

where $P_{k}$ is the state variance matrix which can be initialized with zeros and $H$ is the measurement matrix.

Figure 3.3 shows an example of the filtering procedure above.
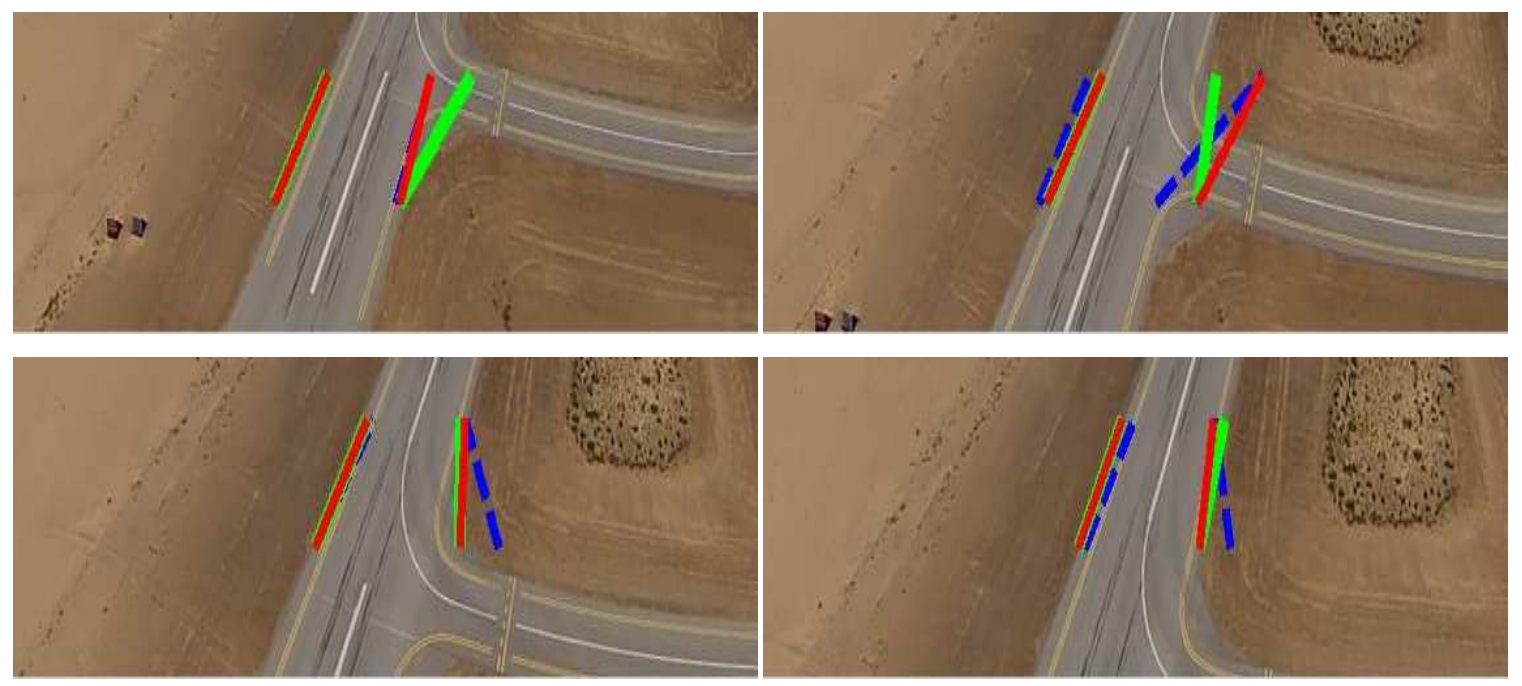

Figure 3.3: Visualization of the Kalman Filter performance in tracking the runway edges. We consider on this picture four consecutive time steps (Left to right, and up to down). Blue line: predicted runway edge locations (from previous step). Green line: runway edge locations (measurements) using vision based segmentation. Red lines: estimation (output of the Kalman filter). 


\section{Chapter 4}

\section{Scale Sensitivity}

An important parameter of the runway edge detection and tracking algorithms presented in this work is the scale parameter. Which scale should be used, and how robust would this choice be to experimental landing conditions?! On the two extremes, a small scale would be too sensitive to image noise, while a large scale may be unable to capture the location of the runway. If no altitude or positional information is available to the UAV, the scale of the runway (on the image) is unknown. If this information is available, one can specify the approximate scale of the runway, which would facilitate the detection of the runway. The area of the neighborhood included in the statistical analysis presented in section 2.2 is also another difficulty, since increasing this area could lead to the inclusion of outliers or clutter located around the edges of the runway. This could lead to another line being falsely detected as a runway edge (false positive), which is undesirable.

We ran multiple experiments over ten representative frames including different views of the lane. Accordingly, there should be a scale by which the edges can be easily and accurately detected and correctly chosen to be part of the lane. This is considered as true positive result. Running an experiment, over ten representative frames of different views of the lane with some clutters in the surrounding over a scale range $S \in[10,15, . ., 75,80]$, and $\theta=45^{\circ}$, gave us a good indicator on how we can

choose the scale of the mask. Hence, the detection process is very sensitive to the 


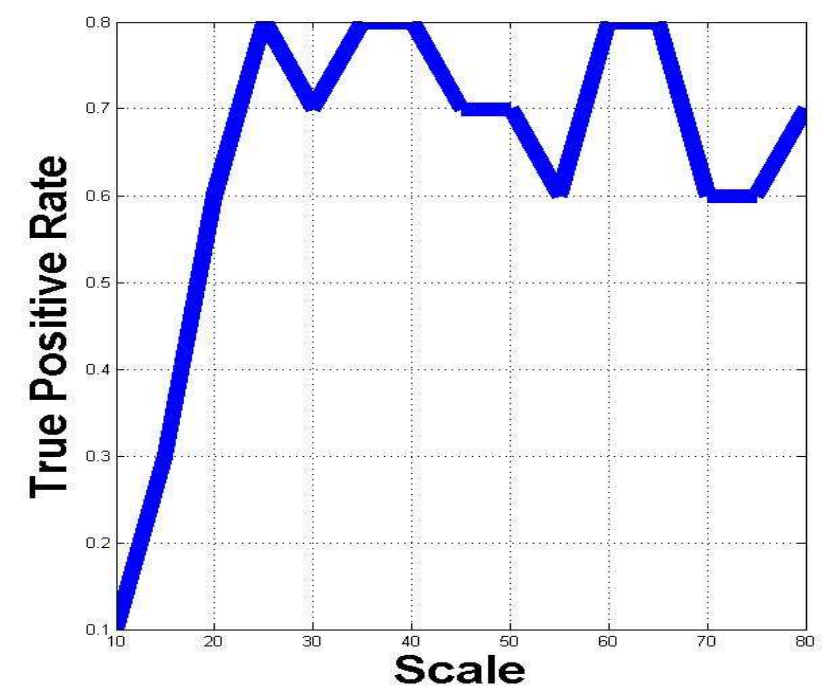

Figure 4.1: True positive rate in function of the scale. This figure shows that the true positive edge detection rate is high on a wide range of scales, which illustrates the robustness of the method with respect to the scale parameter.

scale. Figure 4.1 shows that the best scale is $S=40$. On the other hand, $S=60$ gave the same results, however, it is considered to be big scale. Figure 4.1 shows the true positive rate (TPR) as a function of the chosen scale. 


\section{Chapter 5}

\section{Experiment}

In this chapter, the efficiency and robustness of the detection, segmentation, and tracking algorithms are validated on various video samples. Also, the specifications of the used UAV are mentioned. Moreover, HIL description is provided.

\subsection{Experimental validation}

We evaluated the efficiency of our algorithm over video samples downloaded from a UAV onboard camera filming landing procedures from different altitudes, positions, and lighting conditions. These samples obtained using Saker 4, a medium range UAV developed by the aerospace division of King Abdulaziz City for Science and Technology (KACST). The tested video has a frame resolution of 1920x1080 pixels. The algorithm achieves an average speed of 30 frames per second on this high resolution video. The code is accurate, simple, efficient and supporting real time application. Moreover, we applied the same code under deferent brightness conditions using day and evening video samples to check its robustness.

\subsubsection{Computational Cost}

As mentioned before, the average speed of the algorithm is 30 frames per second. More insight into the speed, the 30 frames per second is the video streaming speed, 
and in order to accommodate such constrain, around $30 \mathrm{~ms}$ per frame is the limit of the processing time. Using our code, the detection step consumes on average $120 \mathrm{~ms}$ per the first frame only. This is not a problem since it gives a very robust initialization, and a number of frames can be skipped over ahead and continue tracking for fast updating.

After detecting the runway in the first frame, the following frames are processed in order to track the runway with average speed 3ms per frame. This means that the algorithm speed is faster ten times than the video streaming speed and hence around 300 frames per second can be processed.

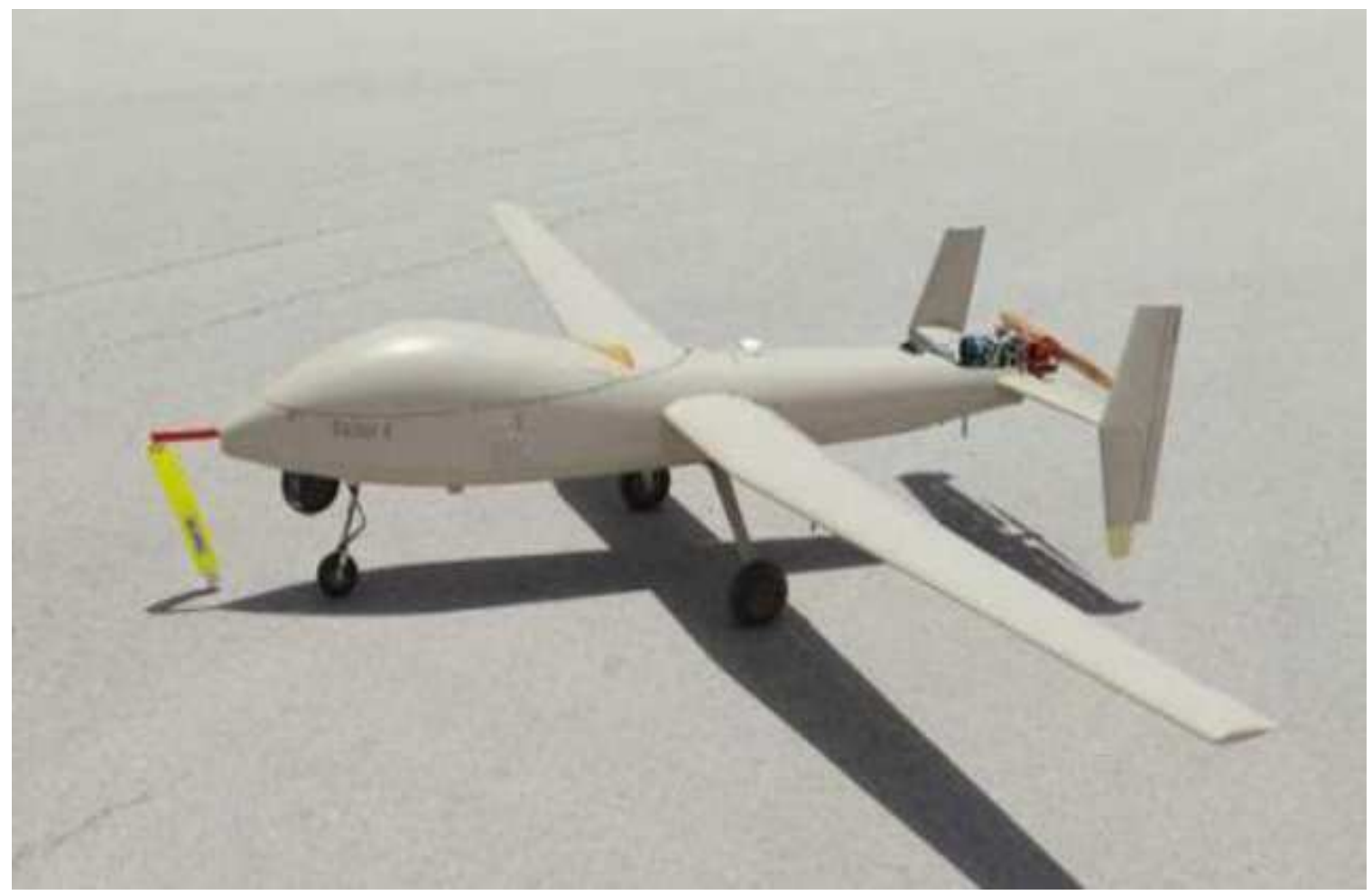

Figure 5.1: Saker $4 \mathrm{UAV}$. This UAV has a maximum takeoff weight of $25 \mathrm{~kg}$ and can carry a payload of $5 \mathrm{~kg}$. With a wingspan of 3.75 meters, it can fly at a maximum speed of $120 \mathrm{kph}$, and a maximal altitude of 5,000 meters[71]. 


\subsubsection{Detection Results}

Figure 5.2 shows the output of sample detection results for a set out of 20 images of the runway taken at different lighting conditions and multiple views of the runway. The results show that our detection algorithm is able to correctly detect the lane in $90 \%$ of the cases, and is thus robust to UAV position and lighting conditions. All detection results were obtained with a fixed scale parameter of $S=40$ (determined empirically from a training set not used in the evaluation).

An important point to emphasize on, which is the geometrical and energy constrains. They are used to avoid the false positives where the algorithm will not give an output such as the images at second row and forth row within the last two columns. This could happen during the detection phase if a delayed or advanced landing command occurred.
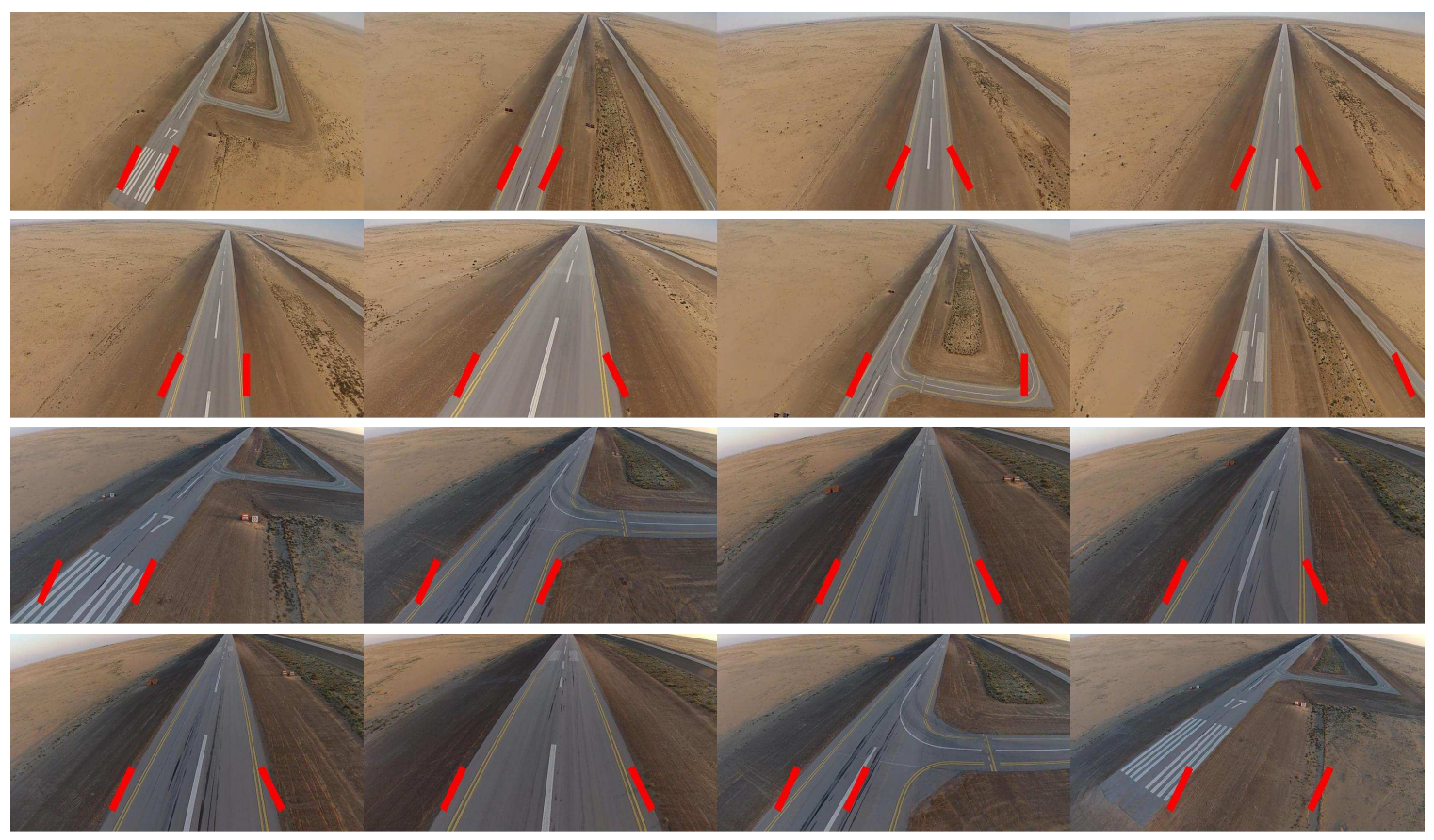

Figure 5.2: Output of the runway detection algorithm. This figure shows the detection output for different views and under different illuminations for two video sequences. 


\subsubsection{Segmentation Results}

The gradient descent evolution is depicted for different types of initialization in Figure 5.3. This shows how robustly the segmentation algorithm can converge towards the runway for any type of initialization around it.

Leveraging KF gives good initialization to the segmentation step in the iterative scheme. This saves time and makes the line segments converge faster to the exact location of the runway using less number of iterations. Also, the time step size can be chosen to be higher and hence converge within a few number of iterations. Also, the terminating step plays a rule since the energy function could have sawtooth behavior while decreasing and hence we can not just look at local minima to terminate the segmentation. Looking at the average energy, backward and forward of a certain number of iterations, at a predefined time instance and evaluating the difference is an efficient a solution.
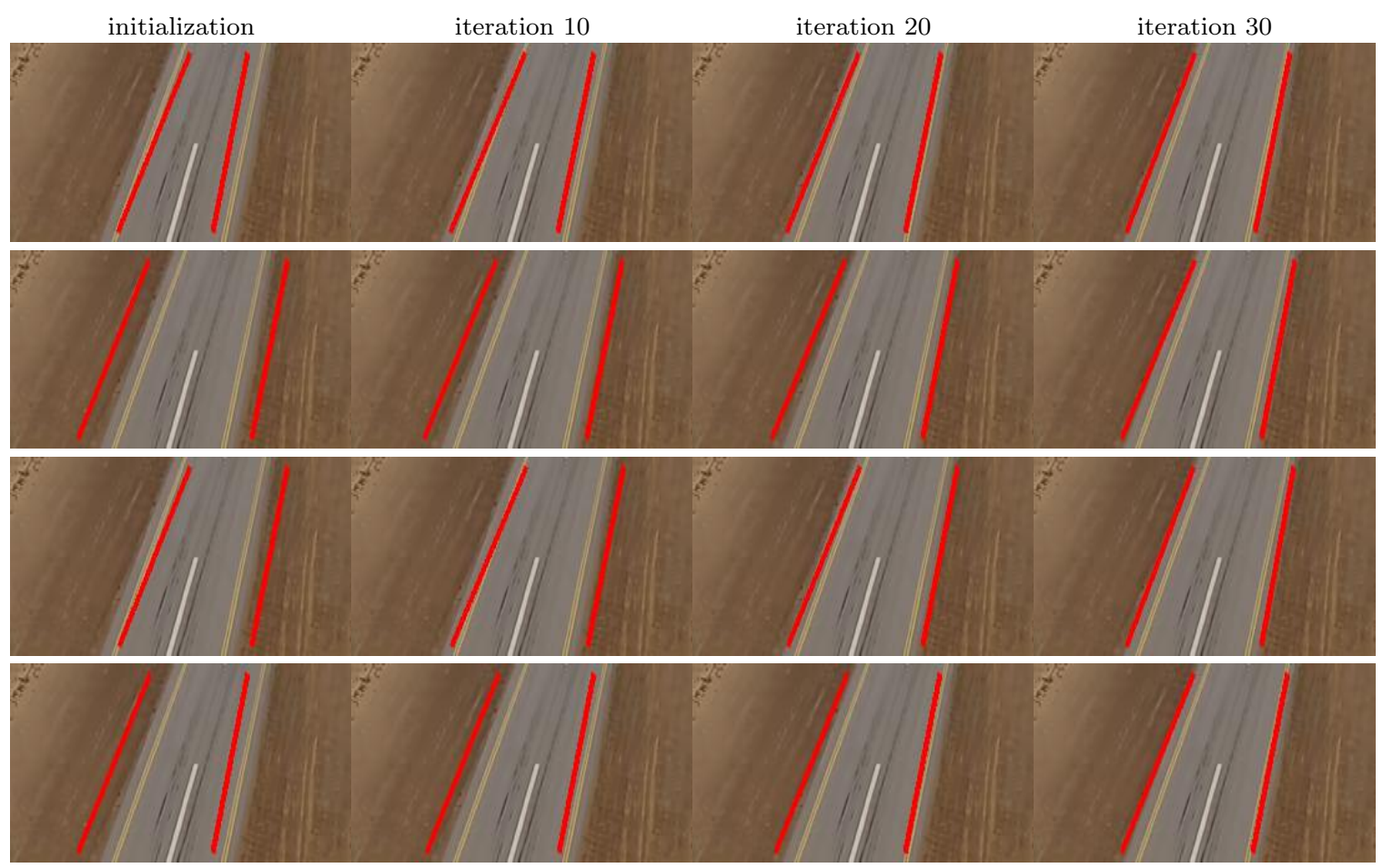

Figure 5.3: GD evolution for different initializations with all possible types of initialization. From the top row to the bottom: outer lines, inner lines, right shift, and left shift, respectively, are the different initializations. 


\subsubsection{Tracking Results}

Figure 5.5 and Figure 5.6 show how the lane is tracked under different light conditions by which robustness is proved. The red lines show the estimated location of the runway using KF over a sequence of images. Reader may find the demonstration videos online [72].

\subsection{Hardware in the loop simulation}

HIL consists of interfacing simulator (a flight simulator) to a sensing and control system (the navigation and control system of a UAV) to evaluate its performance under simulated reality conditions.

In the present case, our HIL framework can be described as the following. The $\mathrm{X}$-Plane flight simulator simulates the view that a UAV in a landing phase would see, and feeds this video data to an embedded computer (TI Beagle Board in the present case). The embedded computer has a ARM processor operating at $1 \mathrm{GHz}$, and 512MB of DDR3 RAM. This computer receives the video data through a HDMI cable (though in reality the video would be acquired by a high resolution video camera), and processes this streaming data using the methods outlined in section 2.2. The resulting edge estimates are sent to an autopilot (currently a Pixhawk autopilot from 3D robotics), which fuses this information with positional and inertial measurements (if available) using the KF outlined in section 3.3 .

We validated the robustness of the algorithm on various video sequences generated by the X-Plane flight simulator. Examples of runway detections in various environmental conditions are illustrated in Figure 5.7, 5.8, and 5.9. The HIL was done in collaboration with my colleague "Wael Alheadary". 

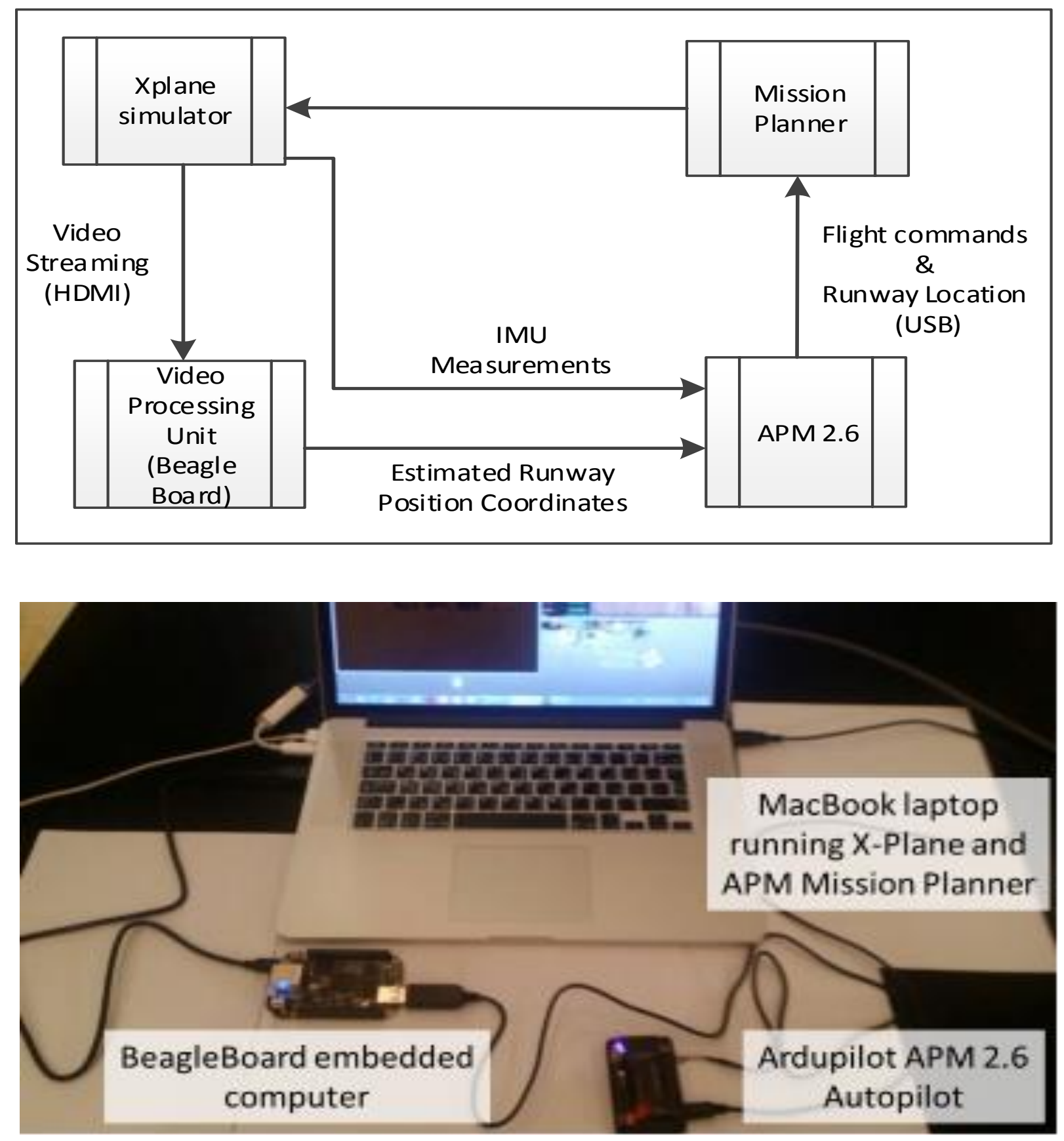

Figure 5.4: Hardware in the Loop simulation system. 

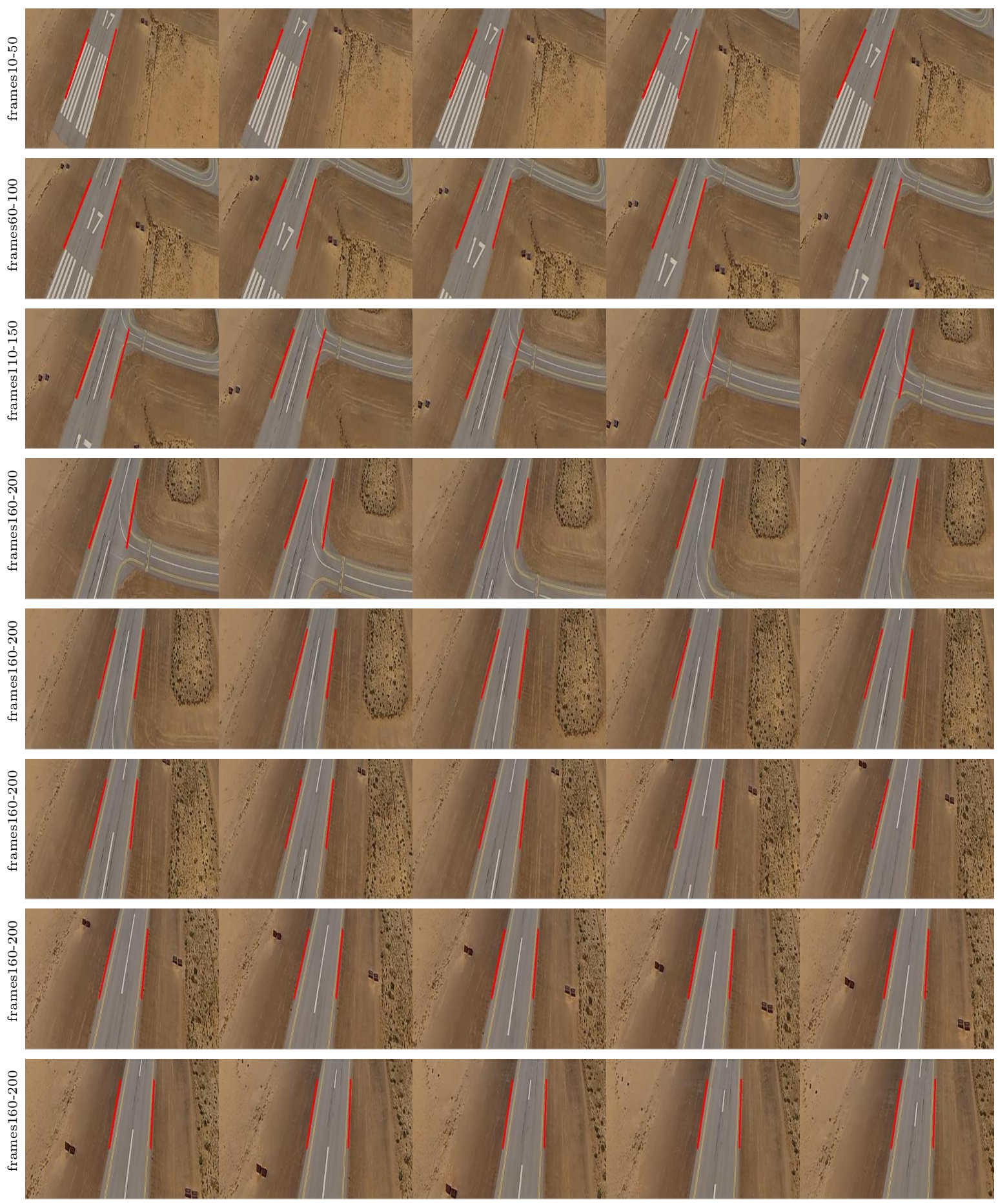

Figure 5.5: Video sequence 1 showing the tracking output. 

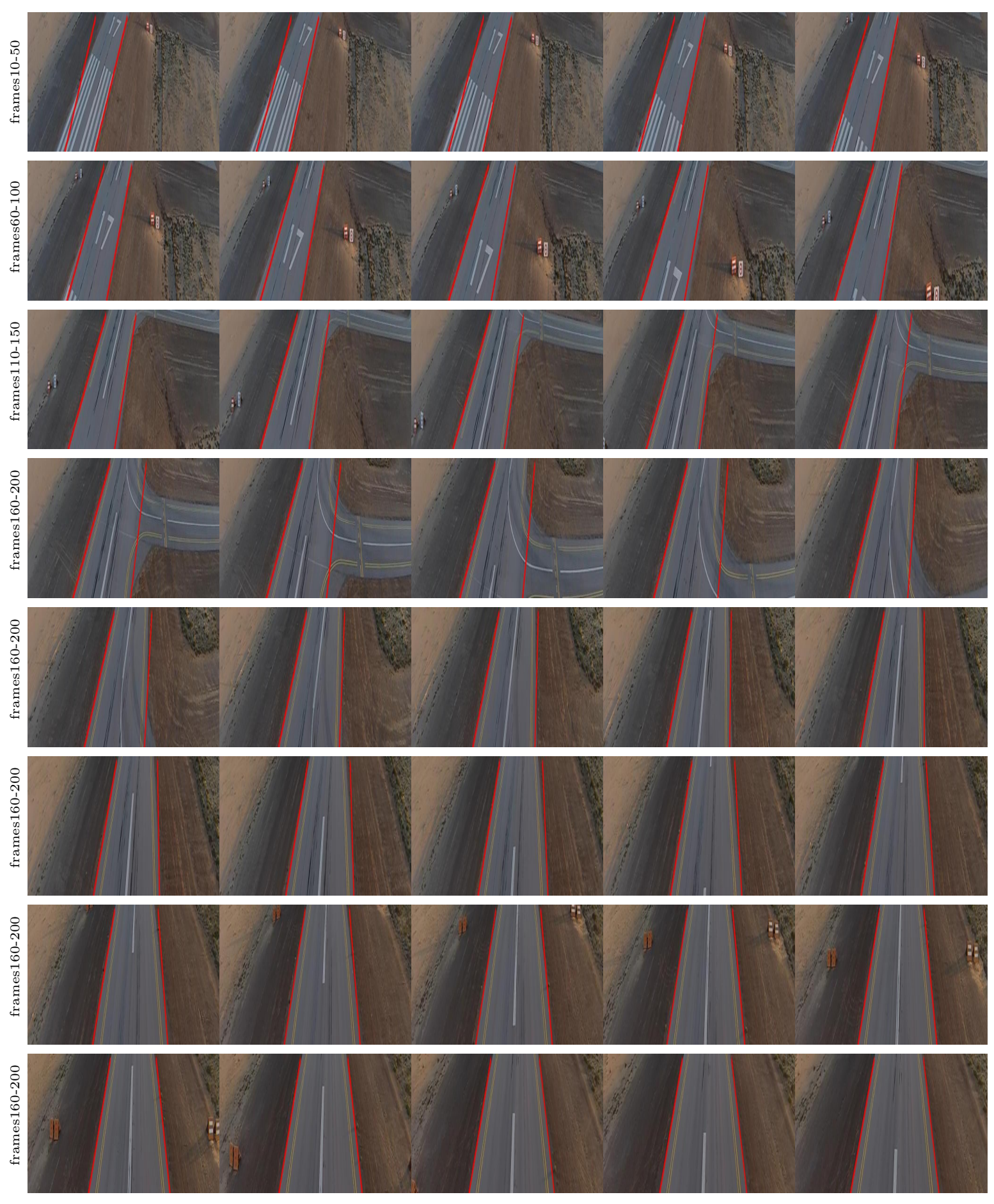

Figure 5.6: Video sequence 2 showing the tracking output. 

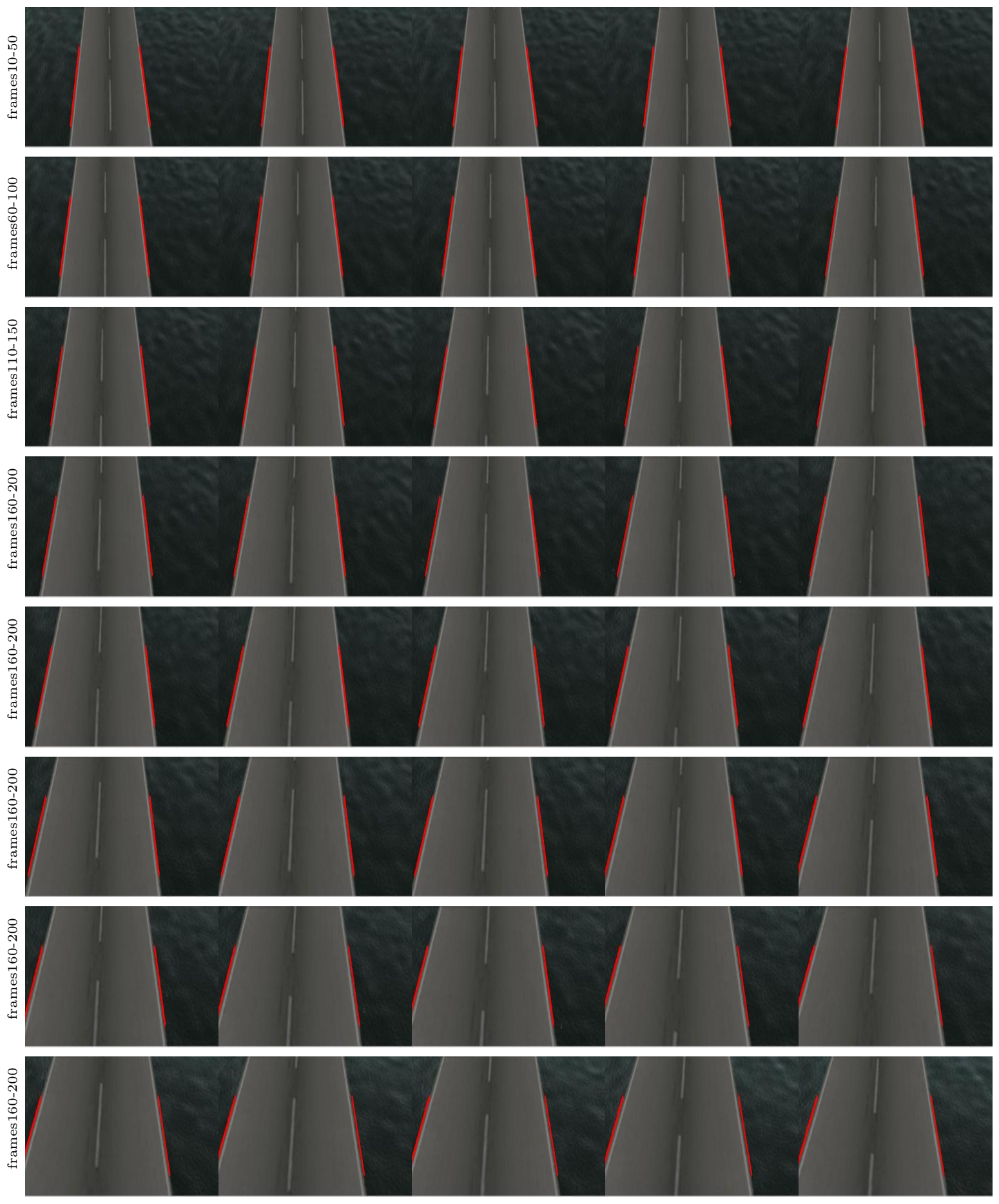

Figure 5.7: Video sequence Xplane 1 showing the tracking output. 

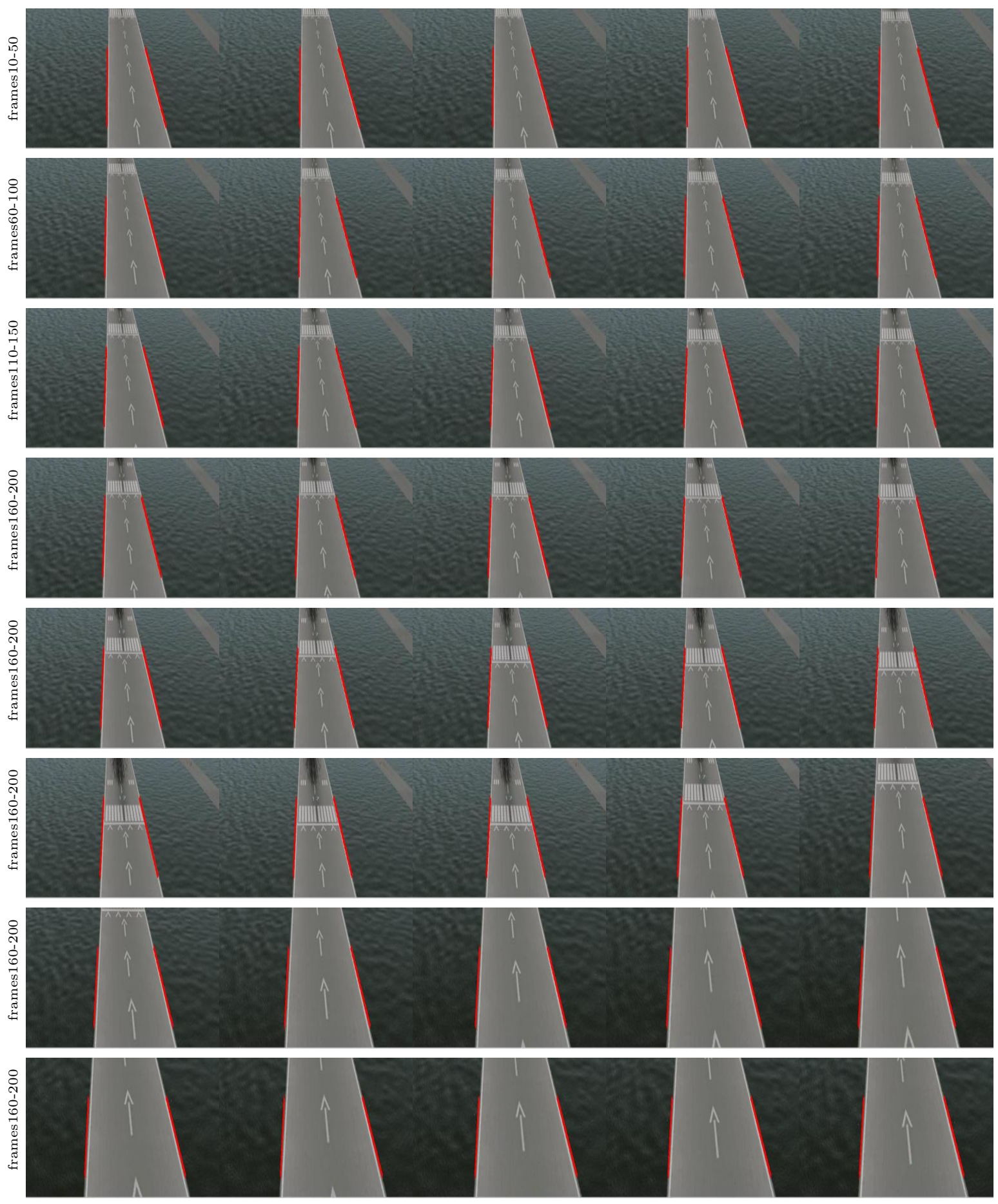

Figure 5.8: Video sequence Xplane 2 showing the tracking output. 

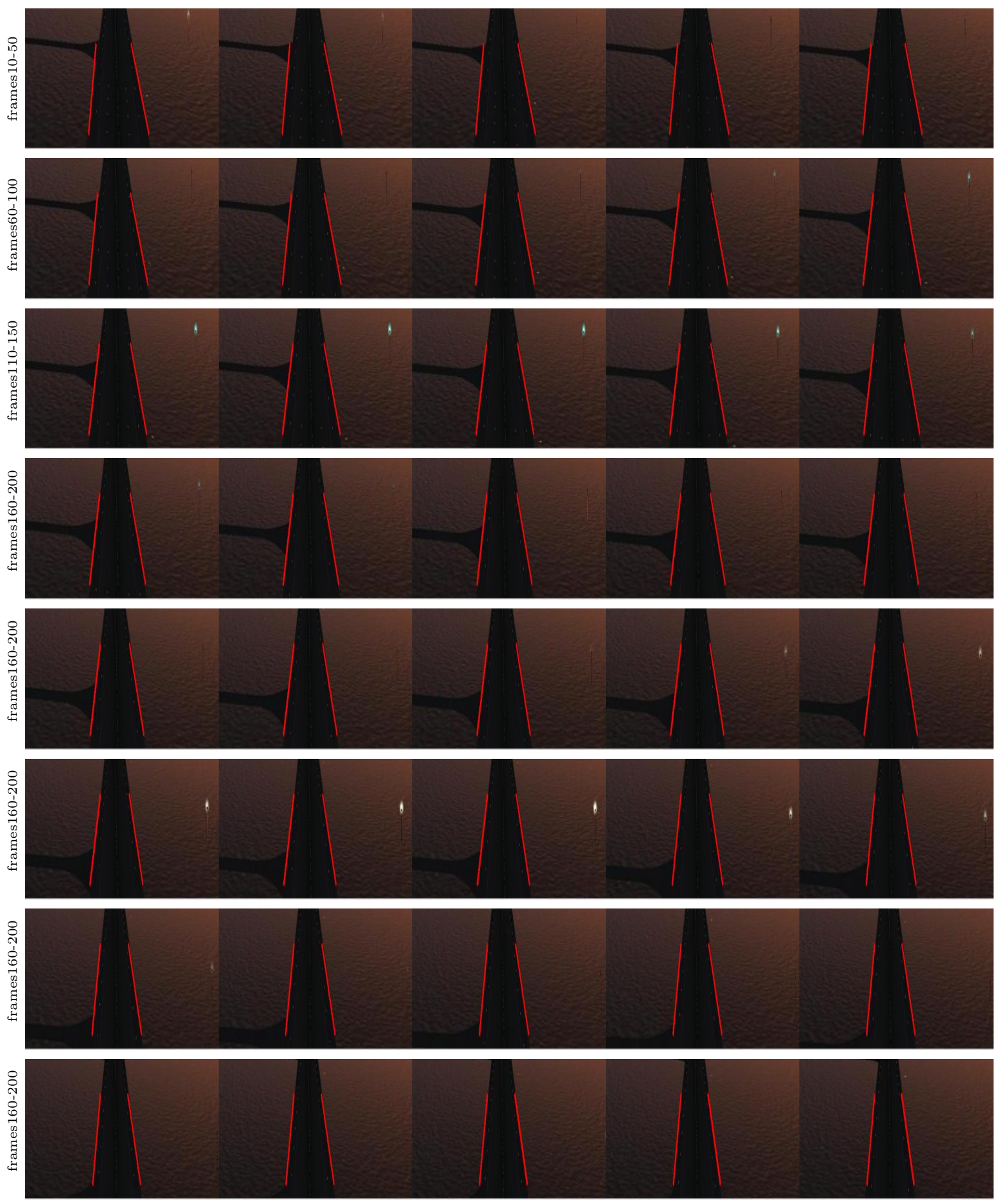

Figure 5.9: Video sequence Xplane 3 showing the tracking output. 


\section{Chapter 6}

\section{Concluding Remarks}

\subsection{Summary}

A fully automatic, robust and real-time runway detection and tracking algorithm for UAV landing applications is presented. Unlike recent approaches to runway detection using computer vision, our approach requires no rendered computer model of the shape and appearance of the runway. This is particularly advantageous since such rendered models are only applicable to a pre-defined runway geometry and under specific lighting and weather conditions. Generating rendered computer models under all possible illumination / weather conditions and runway geometries is not scalable to the wide range of conditions that are observed on a UAV (even under a pre-defined runway). Our approach uses a simple model of the local geometry of the runway and makes no assumption on the appearance of the runway other than dissimilarity to the immediate background. These simple assumptions are more widely applicable to general runways than a specific rendered model, and thus our approach is scalable to realistic conditions encountered by the UAV. We have demonstrated a new model for runways, created an associated optimization problem for the runway, created a robust and efficient coarse-to-fine detection algorithm to localize the runway, and we derived a local optimization approach to refine the results of detection and is used in tracking. The algorithms have been demonstrated experimentally to be robust to a 
wide range of lighting conditions and varied runways, and shown to be real-time. 


\section{REFERENCES}

[1] M. Kontitsis, K. P. Valavanis, and N. Tsourveloudis, "A uav vision system for airborne surveillance," in Robotics and Automation, 2004. Proceedings. ICRA'04. 2004 IEEE International Conference on, vol. 1. IEEE, 2004, pp. 77-83.

[2] G. Medioni, I. Cohen, F. Brémond, S. Hongeng, and R. Nevatia, "Event detection and analysis from video streams," Pattern Analysis and Machine Intelligence, IEEE Transactions on, vol. 23, no. 8, pp. 873-889, 2001.

[3] S. Todorovic and M. C. Nechyba, "Intelligent missions for mavs: visual contexts for control, tracking and recognition," in Robotics and Automation, 2004. Proceedings. ICRA'04. 2004 IEEE International Conference on, vol. 2. IEEE, 2004, pp. $1640-1645$.

[4] A. Ollero, J. Ferruz, F. Caballero, S. Hurtado, and L. Merino, "Motion compensation and object detection for autonomous helicopter visual navigation in the comets system," in Robotics and Automation, 2004. Proceedings. ICRA'04. 2004 IEEE International Conference on, vol. 1. IEEE, 2004, pp. 19-24.

[5] K. Kaaniche, B. Champion, C. Pégard, and P. Vasseur, "A vision algorithm for dynamic detection of moving vehicles with a uav," in Robotics and Automation, 2005. ICRA 2005. Proceedings of the 2005 IEEE International Conference on. IEEE, 2005, pp. 1878-1883.

[6] S. G. Fowers, D.-J. Lee, B. J. Tippetts, K. D. Lillywhite, A. W. Dennis, and J. K. Archibald, "Vision aided stabilization and the development of a quad-rotor micro uav," in Computational Intelligence in Robotics and Automation, $200 \%$. CIRA 200\%. International Symposium on. Ieee, 2007, pp. 143-148.

[7] S. R. Herwitz, L. F. Johnson, J. Arvesen, R. Higgins, J. Leung, and S. Dunagan, "Precision agriculture as a commercial application for solar-powered unmanned 
aerial vehicles," in AIAA 1st Technical Conference and Workshop on Unmanned Aerospace Vehicles, 2002.

[8] D. Hausamann, W. Zirnig, and G. Schreier, "Monitoring of gas transmission pipelines-a customer driven civil uav application," in ODAS Conference, 2003.

[9] P. Van Blyenburgh, "Uavs: an overview," Air 6 S Space Europe, vol. 1, no. 5, pp. 43-47, 1999.

[10] Y. Huang, J. Li, and N. Fan, "Image mosaicing for uav application," in Knowledge Acquisition and Modeling, 2008. KAM'08. International Symposium on. IEEE, 2008, pp. 663-667.

[11] S. Rathinam, Z. Kim, and R. Sengupta, "Vision based following of structures using an uav," Center for Collaborative Control of Unmanned Aerial Vehicles, 2006.

[12] S. Rathinam, P. Almeida, Z. Kim, S. Jackson, A. Tinka, W. Grossman, and R. Sengupta, "Autonomous searching and tracking of a river using an uav," in American Control Conference, 200\%. ACC'0\%. IEEE, 2007, pp. 359-364.

[13] M. Abdelkader, M. Shaqura, C. G. Claudel, and W. Gueaieb, "A uav based system for real time flash flood monitoring in desert environments using lagrangian microsensors," in Unmanned Aircraft Systems (ICUAS), 2013 International Conference on. IEEE, 2013, pp. 25-34.

[14] H. Eisenbeiss, "A mini unmanned aerial vehicle (uav): system overview and image acquisition," International Archives of Photogrammetry. Remote Sensing and Spatial Information Sciences, vol. 36, no. 5/W1, 2004.

[15] S. M. Diamond and M. G. Ceruti, "Application of wireless sensor network to military information integration," in Industrial Informatics, 2007 5th IEEE International Conference on, vol. 1. IEEE, 2007, pp. 317-322.

[16] "Unmanned aerial vehicle systems association," https://www.uavs.org/military. 
[17] D. W. Murphy and J. Cycon, "Applications for mini vtol uav for law enforcement," in Enabling Technologies for Law Enforcement and Security. International Society for Optics and Photonics, 1999, pp. 35-43.

[18] Y.-L. Tang and R. Kasturi, "Runway detection in an image sequence," in ISET/SPIE's Symposium on Electronic Imaging: Science 83 Technology. International Society for Optics and Photonics, 1995, pp. 181-190.

[19] H. Eisenbeiss, "A mini unmanned aerial vehicle (uav): system overview and image acquisition," International Archives of Photogrammetry. Remote Sensing and Spatial Information Sciences, vol. 36, no. 5/W1, 2004.

[20] M. Laiacker, K. Kondak, M. Schwarzbach, and T. Muskardin, "Vision aided automatic landing system for fixed wing uav," in Intelligent Robots and Systems (IROS), 2013 IEEE/RSJ International Conference on. IEEE, 2013, pp. 29712976.

[21] S. R. Herwitz, L. F. Johnson, J. Arvesen, R. Higgins, J. Leung, and S. Dunagan, "Precision agriculture as a commercial application for solar-powered unmanned aerial vehicles," in AIAA 1st Technical Conference and Workshop on Unmanned Aerospace Vehicles, 2002.

[22] S. Rathinam, P. Almeida, Z. Kim, S. Jackson, A. Tinka, W. Grossman, and R. Sengupta, "Autonomous searching and tracking of a river using an uav," in American Control Conference, 2007. ACC'07. IEEE, 2007, pp. 359-364.

[23] F.-B. Hsiao, Y.-H. Chien, T.-L. Liu, M.-T. Lee, W.-Y. Chang, S.-Y. Han, and Y.-H. Wang, "Novel unmanned aerial vehicle system with autonomous flight and auto-lockup capability," in 43rd AIAA Aerospace Sciences Meeting and Exhibit, 2005, pp. 10-13.

[24] D. Hausamann, W. Zirnig, and G. Schreier, "Monitoring of gas transmission pipelines-a customer driven civil uav application," in ODAS Conference, 2003.

[25] D. W. Murphy and J. Cycon, "Applications for mini vtol uav for law enforcement," in Enabling Technologies for Law Enforcement and Security. International Society for Optics and Photonics, 1999, pp. 35-43. 
[26] P. van Blyenburgh, "Uav systems: global review," in Conference, Amsterdam, The Netherlands, 2006.

[27] J. F. Canny, "Finding edges and lines in images," Massachusetts Inst. of Tech. Report, vol. 1, 1983.

[28] P. Arbelaez, M. Maire, C. Fowlkes, and J. Malik, "Contour detection and hierarchical image segmentation," Pattern Analysis and Machine Intelligence, IEEE Transactions on, vol. 33, no. 5, pp. 898-916, 2011.

[29] M. Laiacker, K. Kondak, M. Schwarzbach, and T. Muskardin, "Vision aided automatic landing system for fixed wing uav," in Intelligent Robots and Systems (IROS), 2013 IEEE/RSJ International Conference on. IEEE, 2013, pp. 29712976.

[30] Y. Wang, E. K. Teoh, and D. Shen, "Lane detection and tracking using b-snake," Image and Vision computing, vol. 22, no. 4, pp. 269-280, 2004.

[31] O. Y. Agunbiade, T. Zuva, A. O. Johnson, and K. Zuva, "Enhancement performance of road recognition system of autonomous robots in shadow scenario," arXiv preprint arXiv:1401.2051, 2014.

[32] B. Yang, Y. Wang, and J. Liu, "Lane detection in critical shadow conditions based on double a/d convertors camera," in Artificial Intelligence and Computational Intelligence. Springer, 2011, pp. 54-62.

[33] D. Dusha, W. W. Boles, and R. Walker, "Fixed-wing attitude estimation using computer vision based horizon detection," 2007.

[34] D. Meng, C. Yun-feng, and G. Lin, "A method to recognize and track runway in the image sequences based on template matching," in Systems and Control in Aerospace and Astronautics, 2006. ISSCAA 2006. 1st International Symposium on. IEEE, 2006, pp. 4-pp.

[35] Y. Gui, P. Guo, H. Zhang, Z. Lei, X. Zhou, J. Du, and Q. Yu, "Airborne visionbased navigation method for uav accuracy landing using infrared lamps," Journal of Intelligent 83 Robotic Systems, vol. 72, no. 2, pp. 197-218, 2013. 
[36] P.-s. Ge, L. Guo, G.-k. Xu, R.-h. Zhang, and T. Zhang, "A real-time lane detection algorithm based on intelligent ccd parameters regulation," Discrete Dynamics in Nature and Society, vol. 2012, 2012.

[37] C. Rotaru, T. Graf, and J. Zhang, "Color image segmentation in hsi space for automotive applications," Journal of Real-Time Image Processing, vol. 3, no. 4, pp. 311-322, 2008.

[38] C. Rasmussen, "Combining laser range, color, and texture cues for autonomous road following," in Robotics and Automation, 2002. Proceedings. ICRA'02. IEEE International Conference on, vol. 4. IEEE, 2002, pp. 4320-4325.

[39] Q. Li, N. Zheng, and H. Cheng, "Springrobot: A prototype autonomous vehicle and its algorithms for lane detection," Intelligent Transportation Systems, IEEE Transactions on, vol. 5, no. 4, pp. 300-308, 2004.

[40] Y. He, H. Wang, and B. Zhang, "Color-based road detection in urban traffic scenes," Intelligent Transportation Systems, IEEE Transactions on, vol. 5, no. 4, pp. 309-318, 2004.

[41] J. Alvarez and A. M. Lopez, "Road detection based on illuminant invariance," Intelligent Transportation Systems, IEEE Transactions on, vol. 12, no. 1, pp. 184-193, 2011.

[42] K. Lu, J. Li, X. An, and H. He, "A hierarchical approach for road detection," in Robotics and Automation (ICRA), 2014 IEEE International Conference on. IEEE, 2014, pp. 517-522.

[43] T. M. Jochem and S. Baluja, "A massively parallel road follower," in Computer Architectures for Machine Perception, 1993. Proceedings. IEEE, 1993, pp. 2-12.

[44] J. M. Álvarez, A. M. López, T. Gevers, and F. Lumbreras, "Combining priors, appearance, and context for road detection," IEEE Transactions on Intelligent Transportation Systems, vol. 15, no. 3, pp. 1168-1178, 2014.

[45] W. Phueakjeen, N. Jindapetch, L. Kuburat, and N. Suvanvorn, "A study of the edge detection for road lane," in Electrical Engineering/Electronics, Com- 
puter, Telecommunications and Information Technology (ECTI-CON), 2011 8th International Conference on. IEEE, 2011, pp. 995-998.

[46] W. Liu and S. Li, "An effective lane detection algorithm for structured road in urban," in Intelligent Science and Intelligent Data Engineering. Springer, 2013, pp. $759-767$.

[47] A. H. Lai and N. H. Yung, "Lane detection by orientation and length discrimination," Systems, Man, and Cybernetics, Part B: Cybernetics, IEEE Transactions on, vol. 30, no. 4, pp. 539-548, 2000.

[48] C. Nuthong and T. Charoenpong, "Lane detection using smoothing spline," in Image and Signal Processing (CISP), 2010 3rd International Congress on, vol. 2. IEEE, 2010, pp. 989-993.

[49] J. C. McCall and M. M. Trivedi, "Video-based lane estimation and tracking for driver assistance: survey, system, and evaluation," Intelligent Transportation Systems, IEEE Transactions on, vol. 7, no. 1, pp. 20-37, 2006.

[50] — - "An integrated, robust approach to lane marking detection and lane tracking," in Intelligent Vehicles Symposium. IEEE, 2004, pp. 533-537.

[51] M. Bellino, Y. L. De Meneses, P. Ryser, and J. Jacot, "Lane detection algorithm for an onboard camera," in European Workshop on Photonics in the Automobile. International Society for Optics and Photonics, 2005, pp. 102-111.

[52] A. Lopez, C. Canero, J. Serrat, J. Saludes, F. Lumbreras, and T. Graf, "Detection of lane markings based on ridgeness and ransac," in Intelligent Transportation Systems, 2005. Proceedings. 2005 IEEE. IEEE, 2005, pp. 254-259.

[53] S. Saripalli, J. F. Montgomery, and G. Sukhatme, "Vision-based autonomous landing of an unmanned aerial vehicle," in Robotics and automation, 2002. Proceedings. ICRA'02. IEEE international conference on, vol. 3. IEEE, 2002, pp. 2799-2804.

[54] K. H. Lim, K. P. Seng, L.-M. Ang, and S. W. Chin, "Lane detection and kalmanbased linear-parabolic lane tracking," in Intelligent Human-Machine Systems and 
Cybernetics, 2009. IHMSC'09. International Conference on, vol. 2. IEEE, 2009, pp. 351-354.

[55] C. R. Jung and C. R. Kelber, "Lane following and lane departure using a linearparabolic model," Image and Vision Computing, vol. 23, no. 13, pp. 1192-1202, 2005.

[56] H. Zhang, D. Hou, and Z. Zhou, "A novel lane detection algorithm based on support vector machine," in Progress in Electromagnetics Research Symposium, Hangzhou, China, 2005.

[57] M. Shafique, M. Fahim, and P. Pydipogu, "Robust lane detection and object tracking,in relation to the intelligence transport system," Ph.D. dissertation, School of Engineering, Blekinge Institute of Technology, 2013.

[58] Z. Kim, "Robust lane detection and tracking in challenging scenarios," Intelligent Transportation Systems, IEEE Transactions on, vol. 9, no. 1, pp. 16-26, 2008.

[59] W. Zhu, F. Liu, Z. Li, X. Wang, and S. Zhang, "A vision based lane detection and tracking algorithm in automatic drive," in Computational Intelligence and Industrial Application, 2008. PACIIA'08. Pacific-Asia Workshop on, vol. 1. IEEE, 2008, pp. 799-803.

[60] W. Liu, H. Zhang, B. Duan, H. Yuan, and H. Zhao, "Vision-based real-time lane marking detection and tracking," in Intelligent Transportation Systems, 2008. ITSC 2008. 11th International IEEE Conference on. IEEE, 2008, pp. 49-54.

[61] T. Goldstein, X. Bresson, and S. Osher, "Geometric applications of the split bregman method: segmentation and surface reconstruction," Journal of Scientific Computing, vol. 45, no. 1-3, pp. 272-293, 2010.

[62] A. Sofou, G. Evangelopoulos, and P. Maragos, "Coupled geometric and texture pde-based segmentation," in Image Processing, 2005. ICIP 2005. IEEE International Conference on, vol. 2. IEEE, 2005, pp. II-650. 
[63] P. Kornprobst, R. Deriche, and G. Aubert, Image sequence restoration: A PDE based coupled method for image restoration and motion segmentation. Springer, 1998.

[64] A. Sofou and P. Maragos, "Generalized flooding and multicue pde-based image segmentation," Image Processing, IEEE Transactions on, vol. 17, no. 3, pp. 364376, 2008.

[65] A. Yezzi, A. Tsai, and A. Willsky, "A fully global approach to image segmentation via coupled curve evolution equations," Journal of Visual Communication and Image Representation, vol. 13, no. 1, pp. 195-216, 2002.

[66] S. Boyd and L. Vandenberghe, Convex optimization. Cambridge university press, 2004.

[67] J. Kennedy, "Particle swarm optimization," in Encyclopedia of Machine Learning. Springer, 2010, pp. 760-766.

[68] G. Unal, A. Yezzi, and H. Krim, "Information-theoretic active polygons for unsupervised texture segmentation," International Journal of Computer Vision, vol. 62, no. 3, pp. 199-220, 2005.

[69] S. C. Zhu and A. Yuille, "Region competition: Unifying snakes, region growing, and bayes/mdl for multiband image segmentation," Pattern Analysis and Machine Intelligence, IEEE Transactions on, vol. 18, no. 9, pp. 884-900, 1996.

[70] E. V. Cuevas, "Kalman filter for vision tracking," 2005.

[71] "Unmanned aircraft systems (uas) vision," http://www.uasvision.com/2013/10/ 14/saudi-science-agency-produces-uas/.

[72] K. F. AbuJbara, "Runway detection and tracking algorithm's videos," https: //vimeo.com/user37687230/videos, accessed February 22, 2015.

[73] T. F. Chan and L. A. Vese, "Active contours without edges," Image processing, IEEE transactions on, vol. 10, no. 2, pp. 266-277, 2001. 


\section{APPENDICES}

\section{A Appendix A Chan-Vese Energy Model Optimization - Newton and Gradient Descent}

In our early approach to create our algorithm, we based our research on one of the best types of segmentation which is region competition [69] by minimizing the energy of the regions in the image, hence we can find the boundaries between the regions. The energy function we use tends to divide the image into two regions, (i.e., the runway and the surroundings), which is a generalization of Chan-Vese [73] energy. In our case, we aim to optimize the energy of the vertices of the runway as their locations vary in every frame according to the position and elevation of the UAV. Although we can

minimize the energy for the whole line, we care only about the marked points as we want to optimize the processing time. These points are marked in the following figure.

Let $I: \Omega \rightarrow R$ be an image, $R \subset \Omega$, and $R^{c}=\Omega \backslash R$. We define the energy:

$$
E\left(\left(d_{i}\right)_{i=1}^{4}\right)=\int_{R}(I(x)-u(x))^{2} d x+\int_{R^{c}}(I(x)-v(x))^{2} d x
$$




$$
\begin{gathered}
u(x)=\frac{1}{|R|}\left(\int_{R} I(x) d x\right) \\
v(x)=\frac{1}{\left|R^{c}\right|}\left(\int_{R^{c}} I(x) d x\right)
\end{gathered}
$$

where $u(x), v(x)$ denote the average intensity of the pixels in $R, R^{c}$, respectively, while $\vec{d}$ correspond to the distances between the ends of the lines and the vertices of $\Omega$.

In such optimization problems, the most basic way to solve it is the Gradient Descent. The gradient of $E$ with respect to the boundary $\partial R=c(t)$ is

$$
\nabla E\left(d_{k}\right)=\left(\int_{c(t)}(I(x)-u(x))^{2}-(I(x)-v(x))^{2}\right) N(t) \cdot h(t) d s
$$

where $N(t)$ is the outward normal to $c(t)$ which corresponds to the line curvature; $c(t):[0,1] \rightarrow R^{2}, h(t)$ denotes the perturbation direction of the point, and $\mathrm{t}$ is the parametrization variable. Note that we are shifting the location of the pixel in the horizontal direction only to optimize the process as it is sufficient for identifying the new locations of the pixels.

Then, the points are updated in the direction of gradient descent by

$$
\vec{d}_{k+1}=\vec{d}_{k}-\Delta t \nabla E\left(\vec{d}_{k}\right)
$$

where

$$
\Delta t=\left(\frac{\epsilon}{\|\nabla E\|}\right)
$$

and

$$
\nabla E(\vec{d})=\left[\begin{array}{llll}
\frac{\partial E}{\partial d_{1}} & \frac{\partial E}{\partial d_{2}} & \frac{\partial E}{\partial d_{3}} & \frac{\partial E}{\partial d_{4}}
\end{array}\right]
$$


For instance, at $P_{3}$, we have the following:

$$
\begin{gathered}
c(t)=\left(\begin{array}{l}
x(t) \\
y(t)
\end{array}\right)=\left(\begin{array}{c}
L_{1}-d_{3} \\
0
\end{array}\right)+\left(\begin{array}{c}
\left(L_{1}-d_{4}\right)-\left(L_{1}-d_{3}\right) \\
L_{2}-0
\end{array}\right) t \\
=\left(\begin{array}{c}
L_{1}-d_{3} \\
0
\end{array}\right)+\left(\begin{array}{c}
d_{3}-d_{4} \\
L_{2}
\end{array}\right) t \\
t \in[0,1] \\
h(t)=\frac{\partial c(t)}{\partial d_{3}}=\left(\begin{array}{c}
1-t \\
0
\end{array}\right) \\
c^{\prime}(t)=\left(\begin{array}{c}
d 3-d 4 \\
L_{2}
\end{array}\right) \\
\left|c^{\prime}(t)\right|=\sqrt{\left(d_{3}-d_{4}\right)^{2}+\left(L_{2}\right)^{2}}
\end{gathered}
$$

As defined previously that $N$ is the outward normal to $c(t)$, it can be related to the tangent $T$ to $c(t)$ by $N=J * T$, where $J$ is the rotation matrix with $\theta=-90^{\circ}$ counter clockwise.

$$
\begin{gathered}
J=\left[\begin{array}{cc}
0 & -1 \\
1 & 0
\end{array}\right] \\
T=\frac{c^{\prime}(t)}{\left|c^{\prime}(t)\right|}
\end{gathered}
$$

where $\left|c(t)^{\prime}\right|$ is the speed of the point, $s$ denotes the arc length at $t$, and $L$ is the full 
arc length.

$$
d s=\left|c^{\prime}(t)\right| d t
$$

Collecting the terms of equations (8-13) results in

$$
N(t) \cdot h(t) d s=L_{2}(t-1) d t
$$

Then, the gradient direction at $P_{3}$ can be derived as follows

$$
\begin{aligned}
\frac{\partial E}{\partial d_{3}} & =L_{2} \int_{0}^{1}\left([I(c(t))-u]^{2}-[I(c(t))-u]^{2}\right)(t-1) d t \\
& =L_{2} \int_{0}^{1}([u-v][2 I(c(t))-(u+v)])(t-1) d t \\
& =0.5 L_{2}(u-v)\left[\int_{0}^{1}(t-1) I(c(t)) d t-0.25(u+v)\right]
\end{aligned}
$$

But

$$
\int_{0}^{1}(t-1) I(c(t)) d t=\int_{0}^{L}\left(\frac{s}{L}-1\right) I(c(s)) \cdot \frac{1}{\left|c^{\prime}(t)\right|} d s
$$

Same argument can be done for the rest of the points, and thus

$$
\begin{gathered}
\frac{\partial E}{\partial d_{4}}=0.5 L_{2}(u-v)\left[\int_{0}^{1}(t) I(c(t)) d t-0.25(u+v)\right] \\
\frac{\partial E}{\partial d_{1}}=0.5 L_{2}(u-v)\left[\int_{0}^{1}(t-1) I(c(t)) d t-0.25(u+v)\right] \\
\frac{\partial E}{\partial d_{2}}=0.5 L_{2}(u-v)\left[\int_{0}^{1}(t) I(c(t)) d t-0.25(u+v)\right]
\end{gathered}
$$


Hence, the location of the points is correlated to $\vec{d}$ by the vertices of the rectangular domain $\Omega$.

However, in order to have faster convergence, yet higher computations will be need, Newton step can be used as follows

$$
\begin{gathered}
p=H^{-1} \nabla E \\
d_{k+1}=d_{k}-\Delta t * p
\end{gathered}
$$

where $H_{4 x 4}$ is the hessian matrix whose elements can be computed after redefining the energy equation and the gradient like the following

$$
\begin{gathered}
f(x)=\left((I(x)-u(x))^{2}+(I(x)-v(x))^{2}\right) \\
\frac{\partial E}{\partial d_{i}}=\int_{0}^{1} f(t) h(t) . J c^{\prime}(t) d t
\end{gathered}
$$

Therefor

$$
\frac{\partial^{2} E}{\partial d_{j} d_{i}}=\int_{0}^{1} \frac{\partial f(t)}{\partial d_{j}} h(t) . J c^{\prime}(t) d t+\int_{0}^{1} f(t) h(t) . J \frac{\partial c^{\prime}(t)}{\partial d_{j}} d p
$$

But

$$
h(t) . J \frac{\partial c^{\prime}(t)}{\partial d_{j}}=0
$$

Hence, we see that

$$
\frac{\partial^{2} E}{\partial d_{j} d_{i}}=\int_{0}^{1} \frac{\partial f(t)}{\partial d_{j}} h(t) \cdot J c^{\prime}(t) d t
$$

where $i=1,2$, and $i=3,4$ correspond to curvatures line at the left side and the right side, respectively. While $j=1,2,3,4$ correspond to the four variables that should be 
updated. so,

$$
\begin{aligned}
\frac{\partial f(t)}{\partial d_{j}} & =2\left(\begin{array}{c}
I-u \\
-(I-v)
\end{array}\right) \cdot \frac{\partial}{\partial d_{j}}\left(\begin{array}{c}
I-u \\
-(I-v)
\end{array}\right) \\
& =2\left(\begin{array}{c}
I-u \\
-(I-v)
\end{array}\right) \cdot\left(\begin{array}{c}
\nabla I \cdot \frac{\partial c}{\partial d_{j}}-\frac{\partial u}{\partial d_{j}} \\
\nabla I \cdot \frac{\partial c}{\partial d_{j}}-\frac{\partial v}{\partial d_{j}}
\end{array}\right) \\
& =2\left(\begin{array}{c}
I-u \\
-(I-v)
\end{array}\right) \cdot\left(\begin{array}{c}
\nabla I \cdot h_{j} \cdot \delta_{i, j}^{L}-\frac{\partial u}{\partial d_{j}} \\
\nabla I \cdot h_{j} \cdot \delta_{i, j}^{L}-\frac{\partial v}{\partial d_{j}}
\end{array}\right) \\
& =2\left(\begin{array}{c}
I-u \\
-(I-v)
\end{array}\right) \cdot\left(\begin{array}{l}
I_{x} \cdot h_{j, x} \cdot \delta_{i, j}^{L}-\frac{\partial u}{\partial d_{j}} \\
I_{x} \cdot h_{j, x} \cdot \delta_{i, j}^{L}-\frac{\partial v}{\partial d_{j}}
\end{array}\right)
\end{aligned}
$$

where

$$
\begin{aligned}
& \delta_{i, j}^{L}= \begin{cases}1 & \begin{cases}(L: \text { Left }) & \text { if } j=1,2 \\
(L: \text { Right }) & \text { if } j=3,4\end{cases} \\
0 \quad \text { otherwise }\end{cases} \\
& h_{j}=\left\{\begin{array}{rrr}
\left(\begin{array}{c}
1-t \\
0
\end{array}\right) & \text { if } & j=1,3 \\
\left(\begin{array}{l}
t \\
0
\end{array}\right) & \text { if } & j=1,4
\end{array}\right. \\
& c_{L}(t)=\left\{\begin{array}{rlr}
\left(\begin{array}{c}
d_{1} \\
0
\end{array}\right)+\left(\begin{array}{c}
d_{2}-d_{1} \\
L_{2}
\end{array}\right) t & \text { if } & i=1,2 \\
\left(\begin{array}{c}
L_{1}-d_{3} \\
0
\end{array}\right)+\left(\begin{array}{c}
d_{3}-d_{4} \\
L_{2}
\end{array}\right) t & \text { if } & i=3,4
\end{array}\right.
\end{aligned}
$$

Also, as defined previously, we have

$$
\begin{aligned}
& u(x)=\frac{\int_{R} I(x) d A}{\int_{R} d A} \\
& v(x)=\frac{\int_{R^{c}} I(x) d A}{\int_{R} d A}
\end{aligned}
$$


Now, applying Quotient Rule and Divergence Theorem gives

$$
\begin{aligned}
\frac{\partial u}{\partial d_{j}} & =\frac{1}{A_{R}} \int_{L_{j}}(I-u) h_{j} . N d s \\
\frac{\partial v}{\partial d_{j}} & =\frac{1}{A_{R^{c}}} \int_{L_{j}}(I-v) h_{j} . N d s
\end{aligned}
$$

Then we have the final expression for the second derivative

$$
\begin{aligned}
\frac{\partial^{2} E}{\partial d_{j} d_{i}} & =2 \int_{L_{i}}\left[(I-u-(I-v)) I_{x} \delta_{i, j}^{L} h_{j, x}-\frac{\partial u}{\partial d_{j}}(I-u)-\frac{\partial v}{\partial d_{j}}(I-v)\right] h_{i} . N d s \\
& =2(v-u) \int_{L_{i}} I_{x} \delta_{i, j}^{L} h_{j, x} h_{i} . N d s-2 \frac{1}{A_{R}} \frac{\partial u}{\partial d_{j}} \frac{\partial u}{\partial d_{i}}-2 \frac{1}{A_{R^{c}}} \frac{\partial v}{\partial d_{j}} \frac{\partial v}{\partial d_{i}}
\end{aligned}
$$




\section{B Papers Submitted and Under}

\section{Preparation}

- Khaled Abu Jbara, Wael Alheadary, Ganesh Sundaramorthi, and Christian Claudel, "A Robust Vision-based Runway Detection and Tracking Algorithm for Automatic UAV Landing", Accepted in International Conference on Unmanned Aircraft Systems (ICUAS), Feb. 2015. 